\title{
Remarks on nonlinear relation among phases and frequencies in modulational instabilities of parallel propagating Alfvén waves
}

\author{
Y. Nariyuki and T. Hada \\ Department of Earth System Science and Technology, Kyushu University, 816-8580, Kasuga, Japan \\ Received: 23 March 2006 - Revised: 18 July 2006 - Accepted: 18 July 2006 - Published: 24 August 2006
}

\begin{abstract}
Nonlinear relations among frequencies and phases in modulational instability of circularly polarized Alfvén waves are discussed, within the context of one dimensional, dissipation-less, unforced fluid system. We show that generation of phase coherence is a natural consequence of the modulational instability of Alfvén waves. Furthermore, we quantitatively evaluate intensity of wave-wave interaction by using bi-coherence, and also by computing energy flow among wave modes, and demonstrate that the energy flow is directly related to the phase coherence generation. We first discuss the modulational instability within the derivative nonlinear Schrödinger (DNLS) equation, which is a subset of the HallMHD system including the right- and left-hand polarized, nearly degenerate quasi-parallel Alfvén waves. The dominant nonlinear process within this model is the four wave interaction, in which a quartet of waves in resonance can exchange energy. By numerically time integrating the DNLS equation with periodic boundary conditions, and by evaluating relative phase among the quartet of waves, we show that the phase coherence is generated when the waves exchange energy among the quartet of waves. As a result, coherent structures (solitons) appear in the real space, while in the phase space of the wave frequency and the wave number, the wave power is seen to be distributed around a straight line. The slope of the line corresponds to the propagation speed of the coherent structures. Numerical time integration of the Hall-MHD system with periodic boundary conditions reveals that, wave power of transverse modes and that of longitudinal modes are aligned with a single straight line in the dispersion relation phase space, suggesting that efficient exchange of energy among transverse and longitudinal wave modes is realized in the Hall-MHD. Generation of the longitudinal wave modes violates the assumptions employed in deriving the DNLS such as the quasi-static approximation, and thus
\end{abstract}

Correspondence to: Y. Nariyuki

(nariyuki@esst.kyushu-u.ac.jp) long time evolution of the Alfvén modulational instability in the DNLS and in the Hall-MHD models differs significantly, even though the initial plasma and parent wave parameters are chosen in such a way that the modulational instability is the most dominant instability among various parametric instabilities. One of the most important features which only appears in the Hall-MHD model is the generation of sound waves driven by ponderomotive density fluctuations. We discuss relationship between the dispersion relation, energy exchange among wave modes, and coherence of phases in the waveforms in the real space. Some relevant future issues are discussed as well.

\section{Introduction}

In various areas in space and astrophysical environment, for instance in the solar wind and in foreshock region of planetary bowshocks, parametric instabilities of magnetohydrodynamic (MHD) waves are thought to play essential roles in generating MHD turbulence. Spacecraft observations suggest that, within the MHD turbulence in the solar wind and in the earth's foreshock, a large number of localized structures are often embedded (Mann et al., 1994; Dudok de Wit et al., 1999; Lucek et al., 2004; Tsurutani et al., 2005). Also, recent surrogate data analysis using magnetic field data obtained by Geotail spacecraft (Hada et al., 2003; Koga and Hada, 2003) has revealed that large amplitude MHD waves observed in the earth's foreshock are not completely phase random, but are almost always phase correlated to a certain degree. Furthermore, the larger the MHD wave amplitude, the stronger the wave phase correlation, implying that the detected phase coherence is a consequence of nonlinear interaction among the MHD waves. Since the finite phase coherence in the Fourier space corresponds to the presence of localized structures in the real space, the large amplitude MHD turbulence in the foreshock should be regarded as a superposition

Published by Copernicus GmbH on behalf of the European Geosciences Union and the American Geophysical Union. 
of random phase MHD turbulence plus a large number of localized structures.

Since the MHD waves are dispersive in general, it is natural to infer that the localized structures once produced would disperse away as time elapses. On the other hand, the nonlinearity of the MHD set of equations acts as a source for production of the localized structures. In fact, inspection of a simple model representing interaction of parallel Alfvén wave modes suggests that, the phase coherence is generated whenever there is an exchange of wave energy among wave modes in resonance (Nariyuki and Hada, 2005). Namely, the localized structures are unstable and fade away, but are also continuously born due to intrinsic nonlinearity of the MHD system. Such behavior of the localized structures is typically seen in nonlinear evolution of Alfvén wave parametric instabilities, in particular, in nonlinear evolution of the modulational instability. In a similar context, Nocera and Buti (1996) discussed formation of localized pulses in a nondissipative DNLS system, and Hasegawa et al. (1981) observed emergence of organized structures in the Kortewegde Vries equation. The four-wave interaction scheme was used to explain the emergence of organized structures in the unforced, dissipative DNLS (Krishan and Nocera, 2003). In this paper, we discuss implications of the presence of finite phase correlation, which corresponds to the presence of localized structures, generated by the wave-wave interactions in modulational instability of circularly polarized Alfvén waves, within the context of one dimensional, dissipationless, unforced fluid system. We will emphasize the importance of nonlinear relations among frequencies and phases in identifying relevant physical process involved in the modulational instability.

The plan of the paper is as follows: In Sect. 2, we review and compare the parametric instabilities in the HallMHD system and in the DNLS equation. In Sect. 3, we discuss the relationship between generation of solitary waves and nonlinear wave-wave interaction in detail, by examining numerically produced Alfvénic turbulence using the DNLS equation. Our main discussions on the phase coherence is presented in this section. In Sect. 4, we discuss phase and frequency features in the Hall-MHD system. From the relation between the plasma density and the envelope of the magnetic field, we examine validity of the so-called quasi-static approximation used in the DNLS. When the longitudinal and transverse wave modes have similar phase velocities, they can couple strongly, and as a result the phase coherence can be generated. We summarize the results and discuss some of the fundamental issues presented in the paper in Sect. 5.

\section{Basic equations and analytical study of parametric in- stabilities}

It has long been known that circularly polarized ("parent") Alfvén waves are parametrically unstable to generation of plasma density fluctuations and "daughter" Alfvén waves with the same polarization as the "parent". As early as in the 1960s, it was shown by Galeev and Oraevskii (1963), and Sagdeev and Galeev (1969) that the circularly polarized Alfvén waves are subject to parametric decay instability (see Appendix). Later, Goldstein (1978) and Derby (1978) derived the dispersion relation of the decay instability of the circularly polarized Alfvén waves using ideal MHD equation set. Roles of the dispersion effect was investigated using the Hall-MHD (two-fluid) set of equations by Wong and Goldstein (1986), Longtin and Sonnerup (1986), and Terasawa et al. (1986). The Hall-MHD equations are

$$
\begin{aligned}
\frac{\partial \rho}{\partial t} & =-\nabla \cdot(\rho \mathbf{v}) \\
\frac{\partial \mathbf{v}}{\partial t} & =-\mathbf{v} \cdot \nabla \mathbf{v}-\frac{1}{\rho} \nabla\left(p+\frac{|\mathbf{b}|^{2}}{2}\right)+\frac{1}{\rho}(\mathbf{b} \cdot \nabla) \mathbf{b} \\
\frac{\partial \mathbf{b}}{\partial t} & =\nabla \times(\mathbf{v} \times \mathbf{b})-\nabla \times\left(\frac{1}{\rho}(\nabla \times \mathbf{b}) \times \mathbf{b}\right) \\
\nabla \cdot \mathbf{b} & =0
\end{aligned}
$$

where the density $\rho$ is normalized to the initial uniform density $\rho_{0}$, the magnetic field $\mathbf{b}$ to the background constant field magnitude $b_{0 x}$, the velocity $\mathbf{v}$ to the Alfvén velocity defined by $\rho_{0}$ and $b_{0 x}$, and the pressure $p$ to the ambient magnetic pressure. Time and space are respectively normalized to the reciprocal of the ion cyclotron frequency and the ion inertial length defined using the background quantities.

In this paper, all the physical quantities are assumed to be dependent only on one spatial coordinate $(x)$. Then the governing equations become

$$
\begin{aligned}
& \frac{\partial \rho}{\partial t}=-\frac{\partial}{\partial x}(\rho u) \\
& \frac{\partial u}{\partial t}=-u \frac{\partial u}{\partial x}-\frac{1}{\rho} \frac{\partial}{\partial x}\left(p+\frac{|b|^{2}}{2}\right) \\
& \frac{\partial v}{\partial t}=-u \frac{\partial v}{\partial x}+\frac{1}{\rho} \frac{\partial b}{\partial x} \\
& \frac{\partial b}{\partial t}=-\frac{\partial}{\partial x}\left(u b-v+\frac{i}{\rho} \frac{\partial b}{\partial x}\right)
\end{aligned}
$$

where $b=b_{y}+i b_{z}$ and $v=v_{y}+i v_{z}$ are the complex transverse magnetic field and velocity, respectively, and $u=v_{x}$ is the longitudinal velocity. For simplicity, we assume the equation of state to be isothermal, $p=\beta \rho$, where a constant $\beta$ is the squared normalized sound wave speed.

In the following, linear perturbation analysis is used to discuss parametric instabilities of parallel propagating Alfvén waves, using the DNLS equation (Sect. 2.1) and the HallMHD equations (Sect. 2.2).

\subsection{Linear perturbation analysis of the DNLS system}

By applying a quasi-static approximation in which hydrodynamic nonlinearities and steeping are weak (i.e., variation of 
the plasma density is caused only by magnetic ponderomotive fluctuations), Rogister (1971) derived a kinetic equation describing the long time evolution of the Alfvén waves. Essentially the same equation was subsequently obtained starting from the two-fluid set of equations (Mjølhus, 1974; Mio et al., 1976; Spangler and Sheerin, 1982; Sakai and Sonnerup, 1983; Mjølhus and Hada, 1997). The fluid version, now known as the DNLS, reads

$$
\frac{\partial b}{\partial t}+\alpha \frac{\partial}{\partial x}\left(|b|^{2} b\right)+i \mu \frac{\partial^{2} b}{\partial x^{2}}=0
$$

where normalizations are the same as in Eqs. (1-4), $\alpha / C_{A}=C_{i}^{2} / 4\left(C_{i}^{2}-C_{s}^{2}\right), 2 \mu / C_{A}=c / \omega_{p i}$ is the ion inertia dispersion length, and $C_{A}, C_{i}, C_{S}$ are the Alfvén, intermediate, and sound speeds, respectively. In the DNLS equation, $b=-v$, and

$u=\rho=\frac{|b|^{2}}{2(1-\beta)}$,

where $\beta=C_{S}^{2} / C_{A}^{2}$ (the quasi-static approximation).

The DNLS describes evolution of weakly nonlinear, quasi-parallel propagating, both right- and left-hand polarized Alfvén waves (or "magnetosonic" and "shear Alfvén" waves), which are nearly degenerate. Modulational instability is driven unstable for the left-hand polarized waves when $\beta<1$, and for the right-hand polarized waves when $\beta>1$ (Mjølhus, 1976; Spangler and Sheerin, 1982; Sakai and Sonnerup, 1983), although presence of resonant ions significantly alter the above conditions, especially for $\beta>1$ and moderate to large ion to electron temperature ratio (Rogister, 1971; Mjølhus and Wyller, 1988; Spangler, 1990; Medvedev et al., 1997). The DNLS is known to be integrable under various boundary conditions (Kaup and Newell, 1978; Kawata and Inoue, 1978; Kawata et al., 1980; Chen and Lam, 2004). In this paper, we restrict our discussion to the case $\beta \ll 1$, since only within this regime the use of the fluid version of the DNLS is justified, unless the ion to electron temperature ratio is extremely low.

By re-scaling of the variables, $t \rightarrow \mu t / \alpha^{2}$ and $x \rightarrow \mu x / \alpha$, (9) is reduced as

$$
\frac{\partial b}{\partial t}+\frac{\partial}{\partial x}\left(|b|^{2} b\right)+i \frac{\partial^{2} b}{\partial x^{2}}=0 .
$$

A parallel, circularly polarized, finite amplitude Alfvén wave, $b_{p}=b_{0} \exp \left(i\left(\omega_{0} t-k_{0} x\right)\right)$, where $b_{0}, \omega_{0}$, and $k_{0}$ are real, satisfies (11) with the dispersion relation for the parent wave, $\omega_{0}=b_{0}^{2} k_{0}+k_{0}^{2}$. Now we superpose small fluctuations to $b_{p}$ and write

$b=b_{p}+\sum_{n=-\infty}^{\infty} \epsilon^{|n|} b_{n} \exp \left(i\left(\omega_{n} t-k_{n} x\right)\right)$,

where $k_{n}=k_{0}+n K, \omega_{n}=\omega_{0}+n \Omega$, and $n$ is an integer (except for 0 ). Here, $\Omega$ and $K$ are the frequency and the wave number of longitudinal perturbations (quasi-modes), $u=\rho$. At order of $\epsilon^{| \pm 1|}$, we obtain linearized equations

$\Omega=2 K\left(b_{0}^{2}+k_{0}\right) \pm K \sqrt{K^{2}+2 b_{0}^{2} k_{0}+b_{0}^{4}}$.

If $0<|K|<b_{0} \sqrt{-2 k_{0}-b_{0}^{2}}$, the system exhibits the modulational instability. The wave number corresponding to the maximum growth rate is $K_{\max }=b_{0}\left(-k_{0}-b_{0}^{2} / 2\right)^{1 / 2}$. When the system is modulationally unstable, the frequency of sideband modes is obtained from the real part of (13) as $\omega_{ \pm 1}=2 v_{\phi 0} k_{ \pm 1}-\omega_{0}$, where $v_{\phi 0}=\omega_{0} / k_{0}$. Therefore, the dispersion relation of the sideband modes appears as a straight line, since the dispersion term is cancelled by the nonlinear term. In other words, the nonlinear effect and the dispersion effect are balanced. We will encounter again such a dispersion relation in numerical analysis in Sects. 3 and 4, where more detailed discussions will be given.

\subsection{Linear perturbation analysis of the Hall-MHD equation}

Finite amplitude, circularly polarized Alfvén wave in the form below is an exact solution to Eqs. (5-8),

$b_{p}=b_{0} \exp \left(i\left(\omega_{0} t-k_{0} x\right)\right)$,

$v_{p}=v_{0} \exp \left(i\left(\omega_{0} t-k_{0} x\right)\right)$,

where $v_{0}=-b_{0} / v_{\phi 0}, v_{\phi 0}=\omega_{0} / k_{0}, \rho=1$, and $u=0$, together with the dispersion relation

$\omega_{0}^{2}=k_{0}^{2}\left(1+\omega_{0}\right)$.

Now we add small fluctuations and write

$\rho=1+\frac{1}{2} \sum_{n=1}^{\infty} \epsilon^{|n|} \rho_{n} \exp (i(n \Omega t-n K x))+c . c .$,
$u=0+\frac{1}{2} \sum_{n=1}^{\infty} \epsilon^{|n|} u_{n} \exp (i(n \Omega t-n K x))+c . c .$,
$v=v_{p}+\sum_{n=-\infty}^{\infty} \epsilon^{|n|} v_{n} \exp \left(i\left(\omega_{n} t-k_{n} x\right)\right)$,
$b=b_{p}+\sum_{n=-\infty}^{\infty} \epsilon^{|n|} b_{n} \exp \left(i\left(\omega_{n} t-k_{n} x\right)\right)$,

where $k_{n}=k_{0}+n K, \omega_{n}=\omega_{0}+n \Omega, n$ is an integer, and c.c. represents the complex conjugate. At order of $\epsilon^{| \pm 1|}$, we obtain the following linearized equations

$$
\begin{aligned}
\Omega \rho_{1} & =K u_{1}, \\
\Omega u_{1} & =K b_{0}\left(b_{+}+b_{-}^{*}\right)+\beta K \rho_{1}, \\
\omega_{+} b_{+} & =-k_{+} v_{+}+k_{+} \frac{b_{0} u_{1}}{2}+k_{+}^{2} b_{+}-\frac{b_{0} k_{0} k_{+}}{2} \rho_{1}, \\
\omega_{-} b_{-}^{*} & =-k_{-} v_{-}^{*}+k_{-} \frac{b_{0} u_{1}}{2}+k_{-}^{2} b_{-}^{*}-\frac{b_{0} k_{0} k_{-}}{2} \rho_{1}, \\
\omega_{+} v_{+} & =\frac{k_{0} v_{0}}{2} u_{1}-k_{+} b_{+}+\frac{b_{0} k_{0}}{2} \rho_{1}, \\
\omega_{-} v_{-}^{*} & =\frac{k_{0} v_{0}}{2} u_{1}-k_{-} b_{-}^{*}+\frac{b_{0} k_{0}}{2} \rho_{1},
\end{aligned}
$$




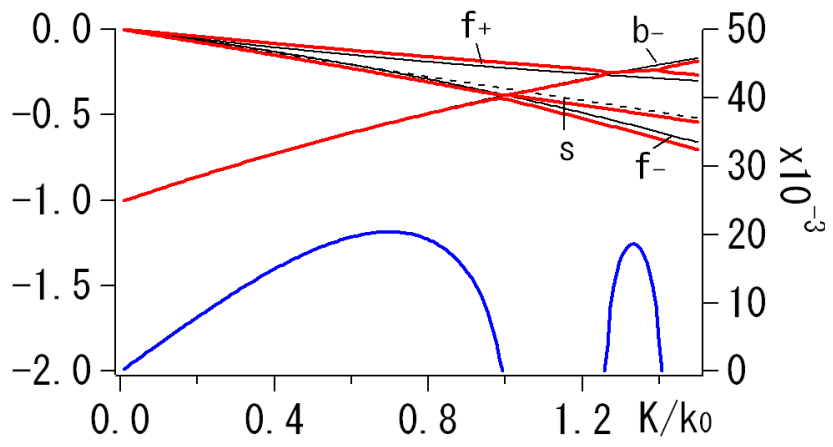

Fig. 1. Dispersion relation of parametric instabilities in the HallMHD system. Shown are the real (red lines, left scale) and imaginary (blue lines, right scale) normalized frequencies plotted versus the normalized wave number, $K / k_{0}$. Parameters used are, $k_{0}=-0.5, \beta=0.5$, and $b_{0}=0.4$. For comparison, the dispersion relation of sideband wave modes and the sound wave (zero parent wave amplitude) is superposed as black solid and dashed lines, respectively. Forward and backward lower (upper) sideband waves are labeled $f-(+)$ and $b-(+)$, and the sound wave is labeled $s$.

where we write the subscripts $( \pm 1)$ as $( \pm)$ for brevity. Combining these equations above, we obtain,

$\left(\Omega^{2}-\beta K^{2}\right) L_{+} L_{-}=\frac{K^{2} b_{0}^{2}}{2}\left(L_{+} R_{-}+L_{-} R_{+}\right)$,

where

$L_{ \pm}=\omega_{ \pm}^{2}-k_{ \pm}^{2}\left(1+\omega_{ \pm}\right)$,

$R_{ \pm}=k_{0} k_{ \pm}\left(\frac{k_{0} \Omega}{\omega_{0} K}+\frac{\Omega \omega_{ \pm}}{k_{0} K}-1-\omega_{ \pm}\right)$.

The dispersion relation (27) has been obtained and solved numerically (Wong and Goldstein, 1986; Longtin and Sonnerup, 1986; Terasawa et al., 1986; Vinas and Goldstein, 1991; Hollweg, 1994; Champeaux et al., 1999). Figure 1 shows the real and imaginary frequencies of the longitudinal waves when $\beta=0.5, b_{0}=0.4$, and $k_{0}=0.5$. Under this particular set of parameters, the modulational instability $\left(0<K / k_{0}<1\right)$ has a growth rate larger than (or almost comparable with) the beat instability around $k=1.3$.

It is instructive to look at Eqs. (23) and (24) in order to discuss how the side band mode frequencies are determined. The r.h.s. of these equations represent three basic processes which determine the transverse magnetic field; the linear response $\left(k_{ \pm} v_{ \pm}^{(*)}\right)$, the $\mathbf{v} \times \mathbf{b}$ nonlinearity $\left(k_{ \pm} b_{0} u_{1} / 2\right)$, and the Hall effect $\left(k_{ \pm}^{2} b_{ \pm}^{(*)}-b_{0} k_{0} k_{ \pm} \rho / 2\right)$. Hereafter we will call these terms as "linear", "nonlinear", and the "dispersive" terms, respectively. For a given set of parent wave parameters ( $b_{0}$ and $\left.k_{0}\right), \beta$, and $K$, one can solve Eq. (27) to find $\Omega$ associated with the modulational instability (eigenvalue), together with ratios among variables, $\rho_{1}, u_{1}, b_{ \pm}^{(*)}$, and $v_{ \pm}^{(*)}$

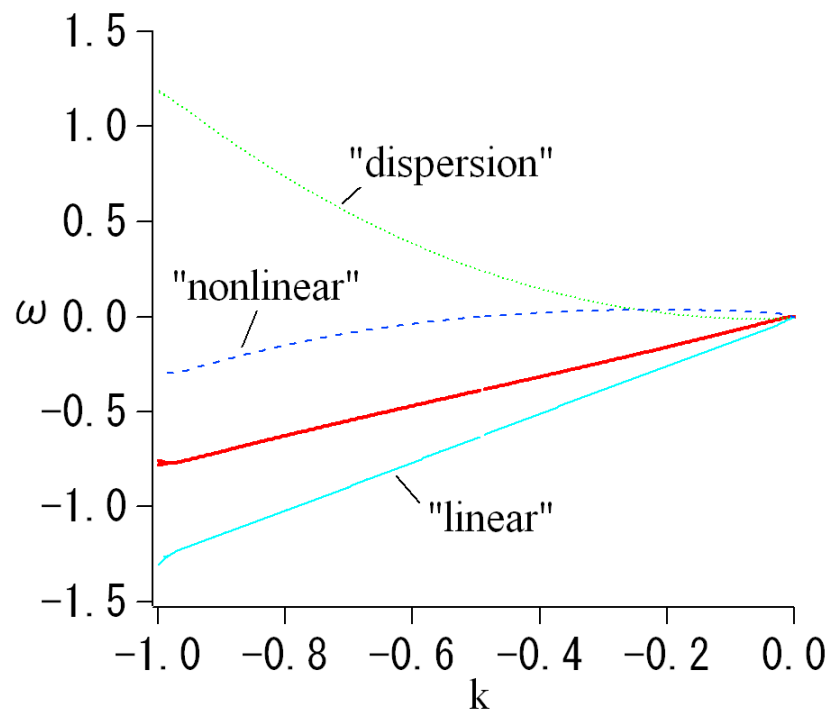

Fig. 2. The three frequency contributions, $\omega_{\text {lin }}, \omega_{n l}$, and $\omega_{\text {disp }}$, plotted versus $k$, using the same set of parameters used for Fig. 1. See text for explanation of how to make the plot. Both $\operatorname{Re}[\omega]$ (red solid line) and $\omega_{\text {lin }}$ are linearly dependent on $k$, and so should be the sum of $\omega_{\text {disp }}$ and $\omega_{n l}$, suggesting that the dispersion and the nonlinear effects are cancelled each other.

(eigenvector). By varying $K$ in both positive and negative regimes, the dispersion relation can be visualized by plotting the real part of $\omega \equiv \omega_{0}+\Omega$ as a function of $k \equiv k_{0}+K$, as shown in Fig. 2 as a solid red line. Superposed in the figure are the plots of $\omega_{\text {lin }}=\left(k_{ \pm} v_{ \pm}^{(*)}\right) / b_{ \pm}^{(*)}, \omega_{n l}=k_{ \pm} b_{0} u_{1} / 2 b_{ \pm}^{(*)}$, and $\omega_{\text {disp }}=k_{ \pm}^{2}-b_{0} k_{0} k_{ \pm} \rho / 2 b_{ \pm}^{(*)}$ versus $k$, which are frequency contribution from the three effects introduced above. By definition, $\omega=\omega_{\text {lin }}+\omega_{n l}+\omega_{\text {disp }}$.

From the figure we notice that both $\omega$ and $\omega_{\text {lin }}$ almost linearly depend on $k$, and thus sum of $\omega_{n l}$ and $\omega_{\text {disp }}$ should also depend linearly on $k$, suggesting that the nonlinear effect and the dispersion effect are balanced, as they were for the DNLS equation discussed previously. In other words, the mismatch of the resonance condition due to the dispersion effect is reduced by nonlinear interaction among the Fourier modes, just like in the case of the DNLS (Sect. 2.1).

\section{Phase coherence in the DNLS equation}

In this section, we discuss the relationship between generation of solitary waves and the wave-wave interaction. In Fig. 3 we show numerical time integration of (11) under periodic boundary conditions, where the envelope $(|b|)$ is plotted in the phase space of time $(t)$ and space $(x)$. As initial conditions, finite amplitude, left-hand polarized, monochromatic Alfvén waves with the wave amplitude $b_{0}=0.4$ and the wave mode number $m=m_{0}=-11$ are given, superposed with a 


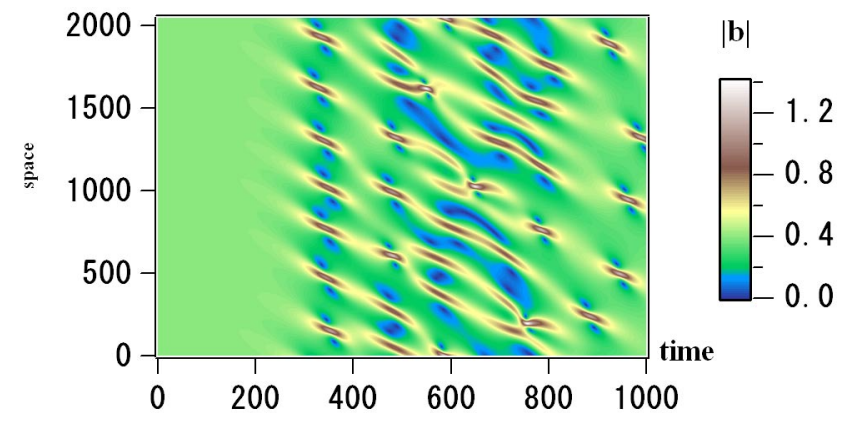

Fig. 3. Time evolution of envelope $|b|$ in the DNLS model (11) with periodic boundary conditions. Initial conditions are given as a superposition of finite amplitude, left-hand polarized monochromatic waves, and very small amplitude white noise.

very small amplitude white noise with $<\left|b_{\text {noise }}\right|^{2}>^{1 / 2}=10^{-5}$ within the range of $-256<m<256$, where the bracket denotes spatial average over the simulation system. In the above, the wave number $k$ is related to the mode number $m$ as $k=2 \pi m / L$, where the system size is $L=256$. We have used the convention that $m$ and $k$ positive (negative) represents the right- (left-) hand polarized waves. For the numerical computation, we have employed the rationalized RungeKutta scheme for time integration and the spectral method for evaluating spatial derivatives. Number of grid points used for this run is 2048 .

Since in Fig. 3 we are plotting the magnetic field envelope, which is constant for the monochromatic, circularly polarized waves, at the beginning not much wave activities are apparent. However, starting from $t \sim 200$, modulation of the envelope becomes increasingly more evident, and around $t \sim 300$ a series of solitary waves is created. The number of solitary waves is decided by the wave number that has the largest growth rate in Eq. (13). After $t \sim 300$ we observe a complex behavior of solitary waves: they appear, propagate, disappear, and interact with each other.

Corresponding time evolution of the power spectrum is shown in Fig. 4. During $0<t \lesssim 200$, the parent wave energy $(m=-11)$ is gradually transferred to the side-band daughter waves (mainly, $m=-4$ and $m=-18$ ) through the modulational instability. Later on, increasingly more waves at different mode numbers are generated due to coupling among finite amplitude waves, and also due to the modulational instability of the daughter waves, as can be seen as widening of the power spectrum in the $m$-space. Around $t \sim 300$, the width of the spectrum is maximized, corresponding to the appearance of the solitary waves. When the solitary waves disappear, the power spectrum becomes narrow again, representing the uncertainty principle, i.e., the width of the wave packet in the real and Fourier space are inversely proportional to each other. We note that solitary waves disappear almost completely at certain time intervals like $t \sim 900$. This is remi-

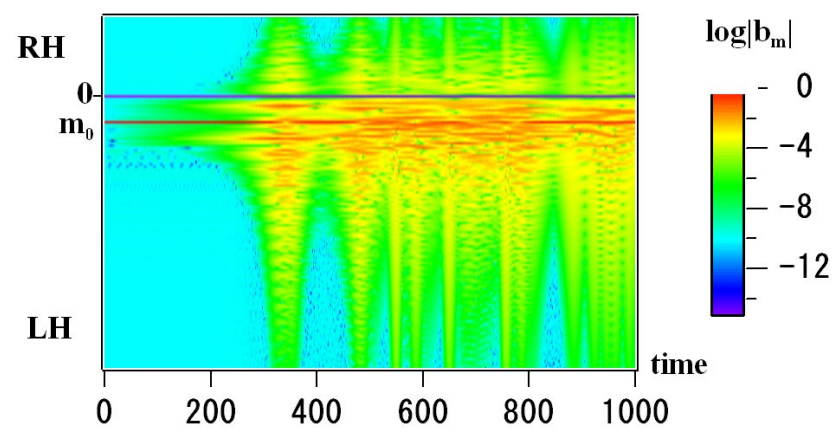

Fig. 4. Time evolution of the power spectrum (in logarithmic scale), $\log \left|b_{m}\right|$, plotted in the phase space of the wave mode number $(m)$ and time. The parent mode is given initially at $m_{0}=-11$. Positive (negative) $m$ corresponds to right- (left-) hand polarized waves. The wave number is given by $k=2 \pi m / L$, where $L=256$ is the system size.

niscent of the presence of (near) recursion of the DNLS equation with periodic boundary conditions.

Next we show that distribution of wave phases and frequencies are closely related to the generation and disappearance of the solitary waves. We write the Fourier transformed magnetic field $b_{k}=\left|b_{k}\right| \exp i \phi_{k}$, and discuss the relation between behavior of solitary waves and correlation among wave phases, $\phi_{k}$. A method to evaluate the wave phase coherence has been proposed (Hada et al., 2003; Koga and Hada, 2003), which we briefly explain below: Suppose we have a data containing waves, $B_{\mathrm{ORG}}(x)$, where "ORG" stands for "original data". In the present analysis, this is a snapshot of simulation data evaluated at certain fixed time. From $B_{\mathrm{ORG}}(x)$ we make a phase randomized surrogate (PRS), $B_{\mathrm{PRS}}(x)$, and a phase correlated surrogate (PCS), $B_{\mathrm{PCS}}(x)$, by shuffling and making equal all the phases, respectively. Then we compute the "phase coherence index",

$C_{\phi}=\left(L_{\mathrm{PRS}}-L_{\mathrm{ORG}}\right) /\left(L_{\mathrm{PRS}}-L_{\mathrm{PCS}}\right)$,

where

$L_{*}=\sum_{x}\left|B_{*}(x+\delta)-B_{*}(x)\right|$

is the first order structure function evaluated for data $(*)$, and the asterisk stands for either ORG, PRS, or PCS. In the above, $\delta$ is an external parameter representing the coarsing scale, which we choose to be the grid size for the present analysis. When $C_{\phi}$ is close to 0 , wave phases are almost random, while $C_{\phi}$ being close to unity suggests that phases are almost completely coherent. Figure 5 shows time evolution of $C_{\phi}$ for the run shown in Fig. 3. Apparently, the appearance and disappearance of solitary wave trains correspond to the increase and decrease of $C_{\phi}$. This is easily understood since the solitary waveform may be produced by equal-phase superposition of many waves with different wavelengths. 


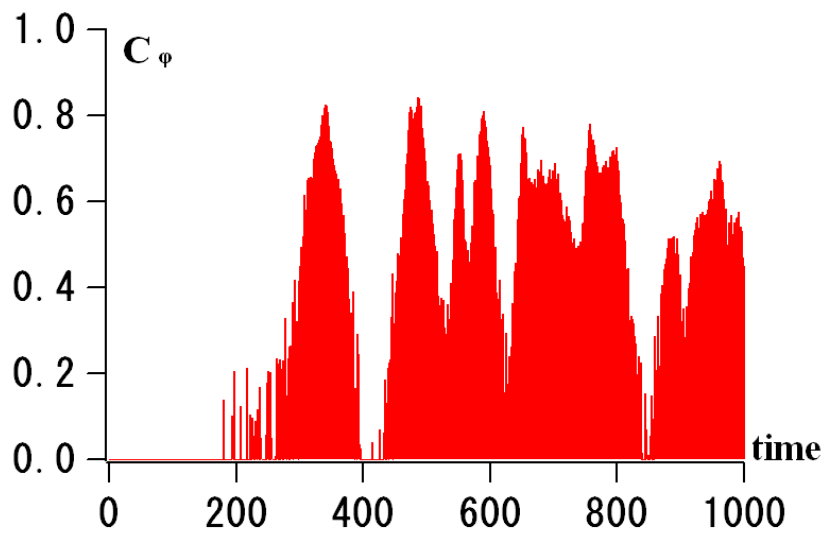

Fig. 5. Time evolution of the phase coherence index, $C_{\phi}$. When $C_{\phi} \sim 0$, the wave phases are almost random, while $C_{\phi} \sim 1$ represents that the wave phases are almost completely correlated.

Now we discuss how phase coherence is generated by the modulational instability in the DNLS. Since the nonlinearity of the DNLS equation is cubic, it follows that, the basic nonlinear interaction in this model is the four wave resonance. (On the other hand, if we regard $|b|^{2}$ as a quasi-mode as represented in the static approximation (10), the interaction can be viewed as the three wave resonance among two Alfvén waves and the quasi-mode.)

We have the resonance relation among the wave numbers,

$k_{1}+k_{2}=k_{3}+k_{4}$,

and a similar relation should hold for the wave frequencies as well. While the resonance condition of the wave frequencies usually has the mismatch due to the dispersion effect in the DNLS, as we saw in Sect. 2, the dispersion effect and the nonlinear effect cancels each other, and so there is no mismatch of frequencies when the wave modes are coupled.

Let us define the relative phase among the four waves as

$\theta(\mathbf{k}) \equiv \phi_{k 1}+\phi_{k 2}-\phi_{k 3}-\phi_{k 4}$,

where $\mathbf{k}=\left\{k_{1}, k_{2}, k_{3}, k_{4}\right\}$ represents the quartets of wave modes in resonance. Small temporal change of $\theta(\mathbf{k})$ implies that the phase coherence between the four waves is strong (locked), because this temporal change corresponds to the mismatch of the resonance condition of frequencies.

From Eqs. (11) and (33), we have

$\frac{d}{d t}\left(\frac{\left|b_{k 1}\right|^{2}}{2}\right)=k_{1} \sum\left|b_{k 1}\right|\left|b_{k 2}\right|\left|b_{k 3}\right|\left|b_{k 4}\right| \sin \theta(\mathbf{k})$,

and

$\frac{d}{d t}\left(\phi_{k 1}\right)=k_{1} \sum \frac{\left|b_{k 2}\right|\left|b_{k 3}\right|\left|b_{k 4}\right|}{\left|b_{k 1}\right|} \cos \theta(\mathbf{k})+k_{1}^{2}$,

where the summation is to be taken over all the combinations of $\left(k_{2}, k_{3}, k_{4}\right)$ which satisfy (32). Equation (34) indicates that

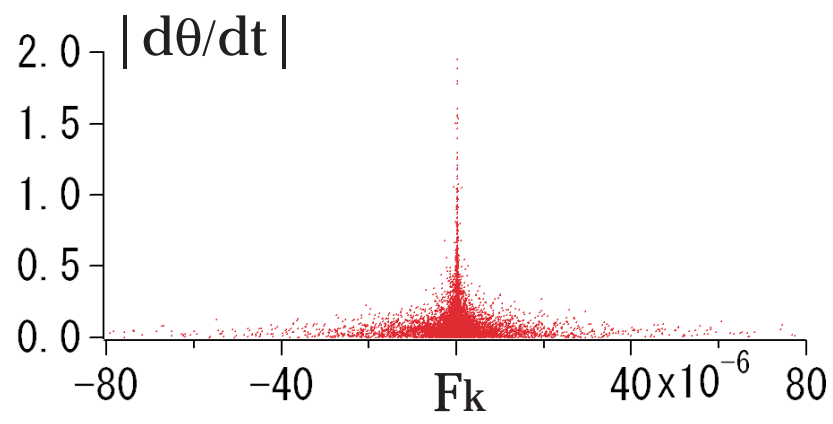

Fig. 6. Relation between $F(\mathbf{k})$ and $|\theta(\mathbf{k})|$ (see text for detailed explanation of the plot and the variables). The set of wave numbers chosen for the plot are $k_{j}=2 \pi m_{j} / L$, where $L=256$ is the system size, and $m_{3}=m_{4}=m_{0}=-11, m_{1}=-15, m_{2}=m_{3}+m_{4}-m_{1}=-7$. The figure suggests that the exchange of wave energy between the sites is enhanced (reduced) when the relative phase is almost constant (varies rapidly) in time. The unit of the vertical axis is degrees per unit time.

the evolution of wave energy of a certain mode is determined by "energy flow" exchanged between the quartets,

$F(\mathbf{k}) \equiv k_{1}\left|b_{k 1}\right|\left|b_{k 2}\right|\left|b_{k 3}\right|\left|b_{k 4}\right| \sin \theta(\mathbf{k})$.

The direction of the energy flow is determined by the sign of $\sin \theta(\mathbf{k})$ and $k_{1}$.

Figure 6 is a scatter plot in the phase space of $F(\mathbf{k})$ and $|d \theta(\mathbf{k}) / d t|(=\mid \theta \dot{(\mathbf{k}}) \mid)$, in which a single dot is plotted at every time step based on the simulation run shown in Fig. 3. The set of wavenumbers, $k_{j}=2 \pi m_{j} / L$, with $m_{3}=m_{4}=m_{0}=-11$, $m_{1}=-15, m_{2}=-7$ are chosen in such a way that the waves satisfy the resonance condition, and also that the modes $m=m_{1}$ and $m_{2}$ are the two daughter waves driven unstable by the parent wave at $m=m_{0}$. It is seen that exchange of the wave energy among the four wave modes is enhanced (reduced) when the relative phase is close to constant (varies rapidly) in time. The same tendency is seen in any quartets in resonance with different choice of the wave numbers. Using this result, we can now interpret the time evolution shown in Figs. 3 and 4 in detail. Around $t \sim 200$, due to the modulational instability driven by the finite amplitude parent wave, a pair of side band waves (daughter waves) appears. At this stage, phase coherence is generated only within a small number of resonant quartets, and the growing sideband waves are restricted within $k<0$ (when $\beta$ is low), because of the derivative nonlinear term in Eq. (11): if a quartet includes some of the right-hand polarized wave modes $(k>0)$, this quartet is "stable" in the sense that the exchange of energy among them stays only at a fluctuation level. Around $t \sim 300$, the sideband waves are sufficiently large, and quartets of higher harmonic sideband wave modes (including $k>0$ modes) become unstable. This broadening of the power spectrum (as seen in Fig. 4) corresponds not only to generation of solitary waves (Fig. 3), but also to the generation of the phase 
coherence (Fig. 5), since a large number of quartes undergo significant nonlinear interaction among them as evidenced in Fig. 6.

The interpretation above is justified by inspection of the dispersion relation directly computed from the simulation run. By computing $\dot{\phi_{k}}$ using (35) and plotting it versus $k_{1}$, the dispersion relation in the simulation system can be visualized for any given time. In Fig. 7 we show time evolution of the dispersion relation, at $t=0, t=200$, and $t=550$. Initially, almost all the wave modes have extremely small amplitude except for the parent wave, and thus they are located around the linear dispersion relation, $\omega=b_{0}^{2} k+k^{2}$. As many of the wave modes acquire finite wave amplitude via the wave-wave interaction, they tend to be aligned on straight lines (two line segments exist in (b): notice a little difference in slopes of lines for $k$ positive and negative). The slope of the line corresponds to the propagation speed of a soliton, and the interval of $k$ which are aligned on the line segment represent the waves the soliton is composed of. At later time (c), it is more evident that the wave modes are concentrated on a number of different line segments, with different ranges of $k$, and with different slopes. These line segments do not necessarily go through the origin, since they represent the four (but not three) wave interaction. Consequently, even though there can be many solitary waves (coherent structures) with different propagation speeds, the resonance condition among the four waves is well satisfied, i.e., the frequency mismatch is small, and thus a large flow of energy exchange among the modes is expected.

The nonlinear interaction among different waves can be inspected through evaluation of the so-called bi-coherence (Dudok de Wit et al., 1999; Diamond et al., 2000),

$b c\left(k_{1}, k_{2}, k_{3}\right)=\frac{\left|<\rho_{k 1} b_{k 2} b_{k 3}^{*}>\right|}{\sqrt{<\left|\rho_{k 1} b_{k 2}\right|^{2}><\left|b_{k 3}\right|^{2}>}}$.

Presence of finite wave power at $k_{1}, k_{2}, k_{3}$, together with $b c \sim 1$ suggests that the waves are in resonance. Figure 8 compares time evolution of averaged bi-coherence index, $b_{\phi}=N^{-1} \sum b c$, total absolute energy flow among all the quartets, $F_{\text {tot }}=N^{-1} \sum|F(\mathbf{k})|$, and the phase coherence index, $C_{\phi}$. On computing $b_{\phi}$ and $F_{\text {tot }}$, all the combinations of wave numbers ( $N$ different ways) are exhausted. Apparently variations of the three quantities agree well to each other, suggesting that the phase coherence is generated as the wave energy is exchanged among resonant quartets.

Finally, we make a remark on the direction of the energy flow. From Fig. 4 we see that the parent wave mode $\left(k=k_{0}=2 \pi m_{0} / L\right)$ remains to be the dominant one throughout the simulation run. Therefore, among the many quartets of wave modes in resonance $\left(k_{1}, k_{2}, k_{3}, k_{4}\right)$ with $k_{1}+k_{2}=k_{3}+k_{4}$, the most dominant quartet (which makes (36) the largest) is the one with $k_{3}=k_{4}=k_{0}$. The resonance condition of wave number for this quartet is,

$k_{0}+k_{0}=k_{1}+k_{2}$.
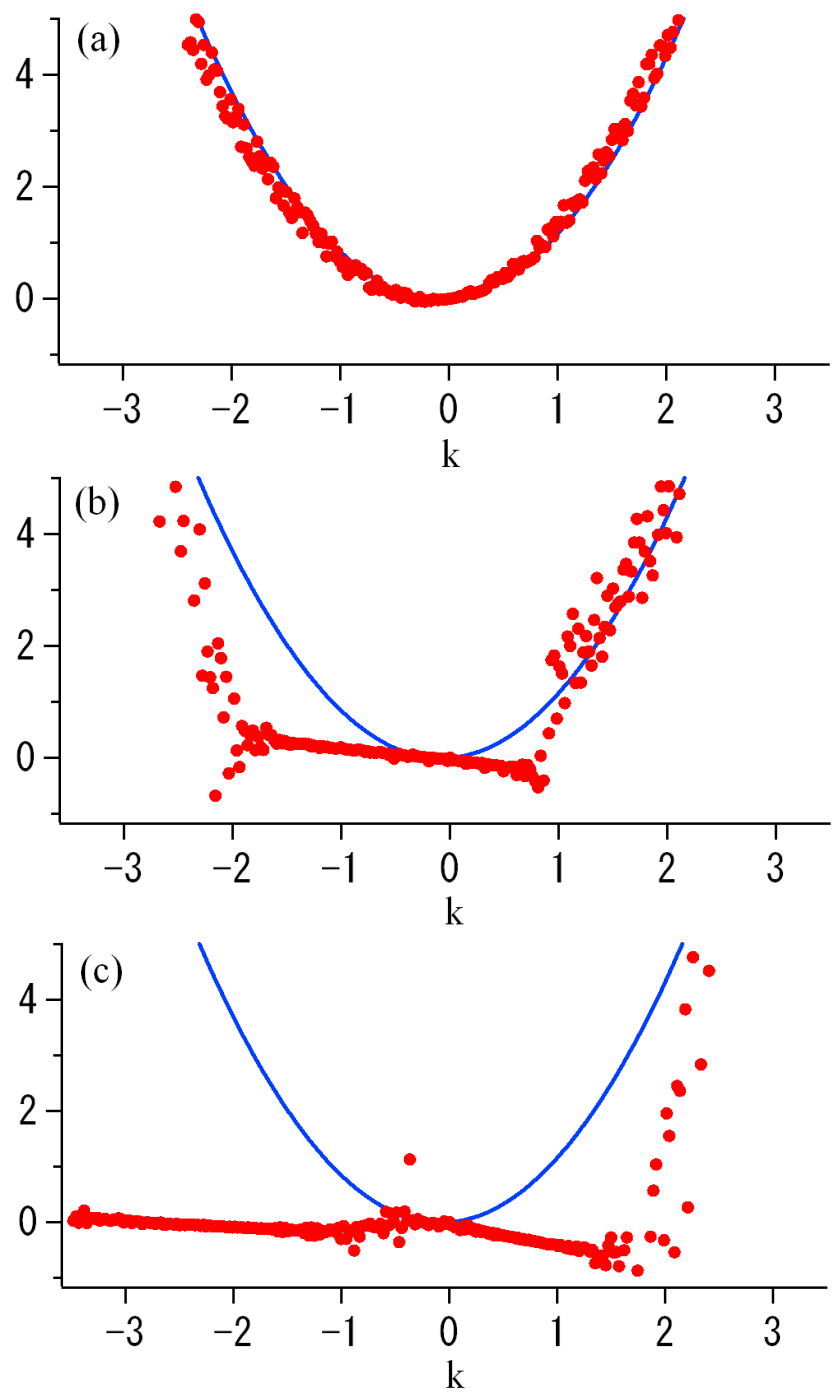

Fig. 7. The wave frequency $\dot{\phi_{k}}$ (red circles) versus $k$ evaluated at (a) $t=0$, (b) $t=350$, and (c) $t=550$. At $t=0$, all the wave modes except for the parent wave have very small wave amplitude, and thus they are located around the linear dispersion relation, $\omega=b_{0}^{2} k+k^{2}$ (blue line). However, as the wave modes grow via wave-wave interaction, they tend to be aligned along straight lines. The slope of the line corresponds to the propagation speed of a soliton, and the interval of $\mathrm{k}$ which are aligned on the line segment represent the waves the soliton is composed of. Among the quartet of waves on a single line, the frequency mismatch for the resonance condition $|\theta(\mathbf{k})|$ is small, and consequently, energy exchange among them $F(\mathbf{k})$ is large, as seen in Fig. 6.

In fact, the set of wave numbers used for Fig. 6 was chosen so that Eq. (38) is satisfied. In Fig. 9 we have plotted $\sin \theta(\mathbf{k})$ in the phase space of $m_{1}$ and time, with $k_{3}=k_{4}=k_{0}$ and $k_{2}$ determined by Eq. (32). The plot shows the direction of the energy flow between the parent wave and the daughter waves. For example, associated with the first formation of 


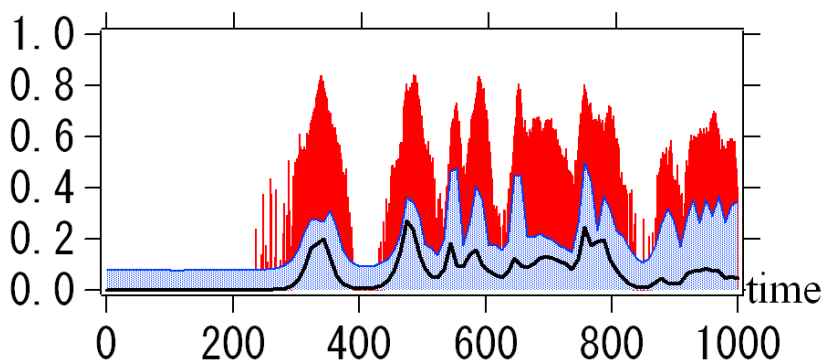

Fig. 8. Time evolution of $C_{\phi}$ (red area), $b_{\phi}$ (blue area), and $F_{\text {tot }} / 10^{-6}$, (black solid line). Variation of $C_{\phi}$ well corresponds to those of $b_{\phi}$ and $F_{\text {tot }}$.

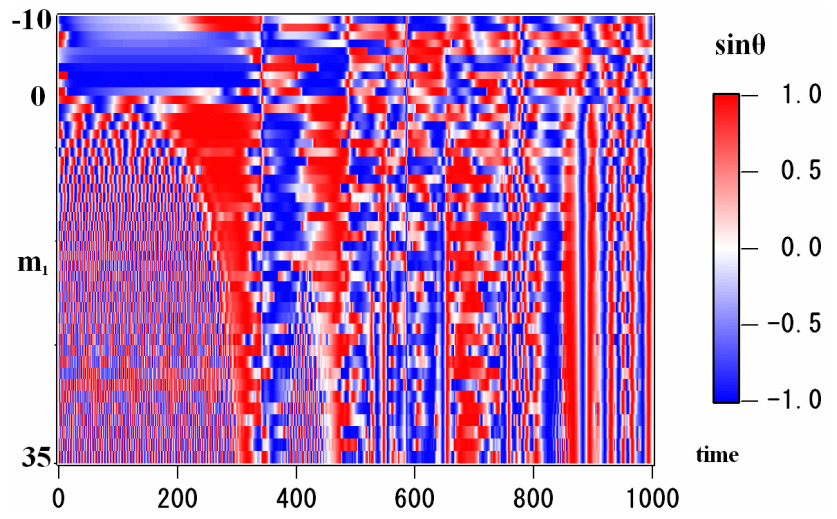

Fig. 9. Evolution of $\sin \theta(\mathbf{k})$, plotted in the phase space of the wave mode number $\left(m_{1}\right)$ and time.

solitary wave rows $(t \sim 200-350), \sin \theta(\mathbf{k})<0$ for $k_{1}<0$ and $\sin \theta(\mathbf{k})>0$ for $k_{1}>0$. Namely, in both regimes of $k_{1}>0$ and $k_{1}<0$, the energy flow defined in Eq. (36) is positive, i.e., the energy is transferred from the parent wave to the daughter waves. During the time interval when solitary waves disappear $(t \sim 350-400)$, we observe the opposite, i.e., the energy flows back from the daughter to the parent waves.

\section{Nonlinear relation among phase and frequency: Hall- MHD equations}

In this section, we discuss the modulational instability in the Hall-MHD equations (Eqs. 5-8), and compare the results with that in the DNLS.

Numerical time integration of the Hall-MHD set of equations, Eqs. (5-8), is performed, in a similar way as for the DNLS equation discussed in the preceding section. As initial conditions, finite amplitude, left-hand polarized, monochromatic, parallel Alfvén waves are given, with the wave amplitude $b_{0}=0.4$ and the mode number $m=m_{0}=-20$. Superposed with the parent wave is a small amplitude white noise

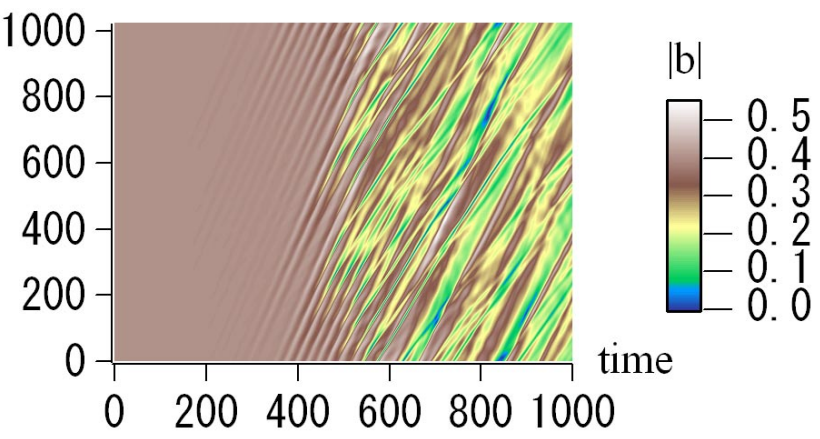

Fig. 10. Time evolution of envelope $|b|$ due to Eqs. (5-8) with periodic boudary conditions. Initial conditions are given as a superposition of finite amplitude, left-hand polarized monochromatic waves, and very small amplitude white noise.

with $<\left|a_{\text {noise }}\right|^{2}>^{1 / 2}=10^{-5}$, where $a$ represents any variable in Eqs. (5-8) within the range of $-128<m<128$. The system size is $L=256$, in which there are 1024 grid points. We chose $\beta=0.5$ so that the modulational instability growth rate is the largest among all the parametric instabilities possibly driven unstable. The parameters specified here are essentially equivalent to the ones used in the previous section.

Figure 10 shows time evolution of $|b|$ plotted in a way similar to Fig. 3, and Fig. 11 represent time evolution of $\log \left|b_{m}\right|$ and $\log \left|\rho_{m}\right|$, which is the power of the transverse and the longitudinal waves, respectively, as a function of the wave mode number, $m$.

From comparison of the DNLS and the Hall-MHD simulation runs, we dicuss validity of various approximations assumed in the DNLS. In particular, we look at the following: (a) the quasi-static approximation, Eq. (10), (b) conservation of the magnetic field energy alone, and (c) the assumption of constant plasma density in the dispersion term (i.e., the Hall-term, $\partial / \partial x\left(\rho^{-1} \partial b / \partial x\right)$, is simplified by assuming $\rho$ to be constant). These approximations are justified as long as the power of the longitudinal modes (Fig. 11b) is much less than that of the transverse modes (Fig. 11a). Around $t \sim 350$, the power spectrum of sideband wave modes and the daughter wave modes begin to grow. To check the validity of the approximation in Eq. (10), we evaluate the cross-correlation function,

$c(\lambda, \tau)=\frac{<|b(x+\lambda, t+\tau)|^{2} \rho(x, t)>}{\sqrt{<|b(x+\lambda, t+\tau)|^{4}><(\rho(x, t))^{2}>}}$

where we simply let $\lambda=\tau=0$ in the present analysis. The result is plotted as a solid line in Fig. 12a. When $0<t<\sim 350$, relation (10) is well held, but around $t \sim 350$, the cross correlation starts to decrease. Figure $12 \mathrm{~b}$ shows time evolution of the magnetic field energy (broken line), parallel kinetic energy (solid line), and perpendicular kinetic energy (dotted line). The decay of the correlation function occurs at the same time as the magnetic field and the perpendicular kinetic 


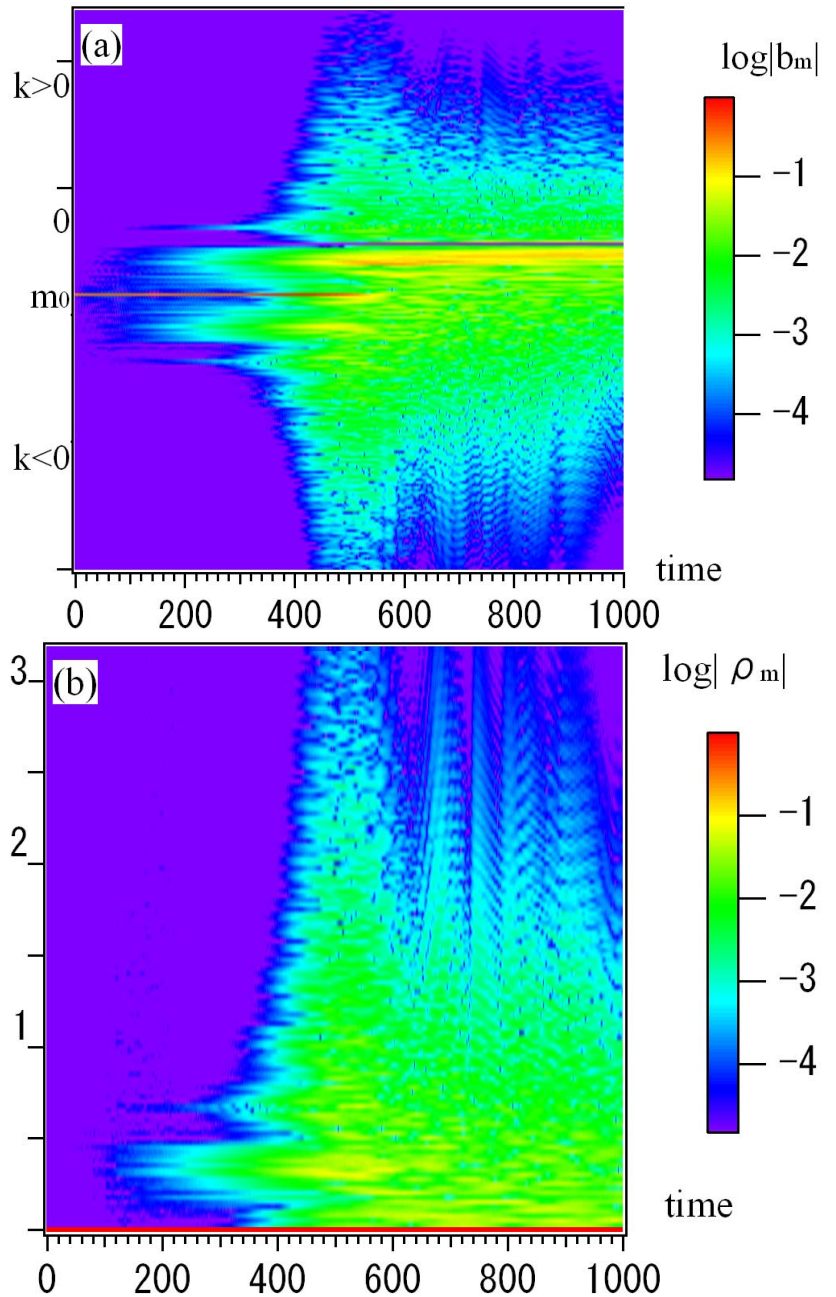

Fig. 11. Time evolution of power spectrum (in logarithmic scale), $\log \left|b_{m}\right|$ and $\log \left|\rho_{m}\right|$, plotted in the phase space of the wave mode number $(m)$ and time. The parent mode is given initially at $m_{0}=-20$. Positive (negative) $m$ corresponds to positive (negative) helicity.

energy both decrease. Around $t \sim 500$, inverse cascade begins to take place (discussion of the inverse cascade in the DNLS can be found in Krishan and Nocera, 2003).

The validity of the static approximation has been examined by several authors. By performing hybrid (kinetic ions + an electron fluid) simulations, Machida et al. (1987) argues that the system automatically adjusts itself to a state described by the static approximation when ion kinetic effects are included. However, as time elapses, longitudinal mode grows and the static approximation is soon violated. Spangler (1987) concluded that the breakdown of the static approximation takes place in association with rapid evolution of wave packets by ponderomotive force. Combining
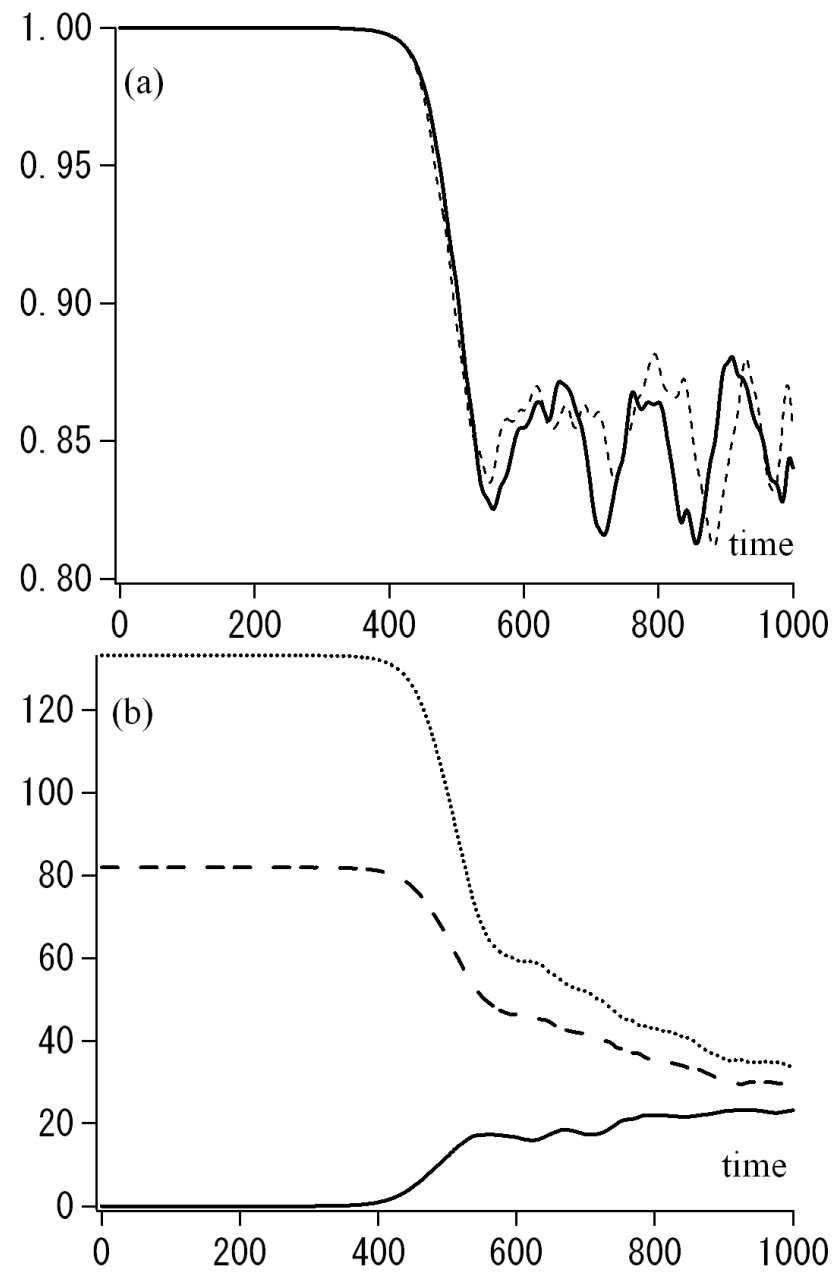

Fig. 12. Time evolution of (a) the cross-correlation, $c(\lambda=0, \tau=0)$ evaluated for the run shown in Fig. 10 (solid line), and $c(0,0)$ evaluated for a run in which the advection term is artificially removed from the Hall-MHD set of equations (broken line). (b) magnetic field energy (broken), perpendicular kinetic energy (dotted), and parallel kinetic energy (solid), plotted versus time. Around $t \sim 350$, together with the decay of the cross-correlation function, the magnetic field and the perpendicular kinetic energy decrease and the parallel kinetic energy increases.

Eqs. (5) and (6), we have

$\frac{\partial^{2} \rho}{\partial t^{2}}-\beta \frac{\partial^{2} \rho}{\partial x^{2}}=\frac{\partial^{2}}{\partial x^{2}}\left(\frac{|b|^{2}}{2}+\rho u^{2}\right)$.

This equation describes propagation of sound waves in a presence of a source term (r.h.s.), which consists of the ponderomotive force, $|b|^{2} / 2$, and the advection effect, $\rho u^{2}$ (Spangler, 1987). The latter represents the "self-coupling" of longitudinal waves.

In order to clarify the roles of the "self-coupling" term, which is not discussed in the analysis of Spangler (1987), we have run numerical experiment in which the advection terms 

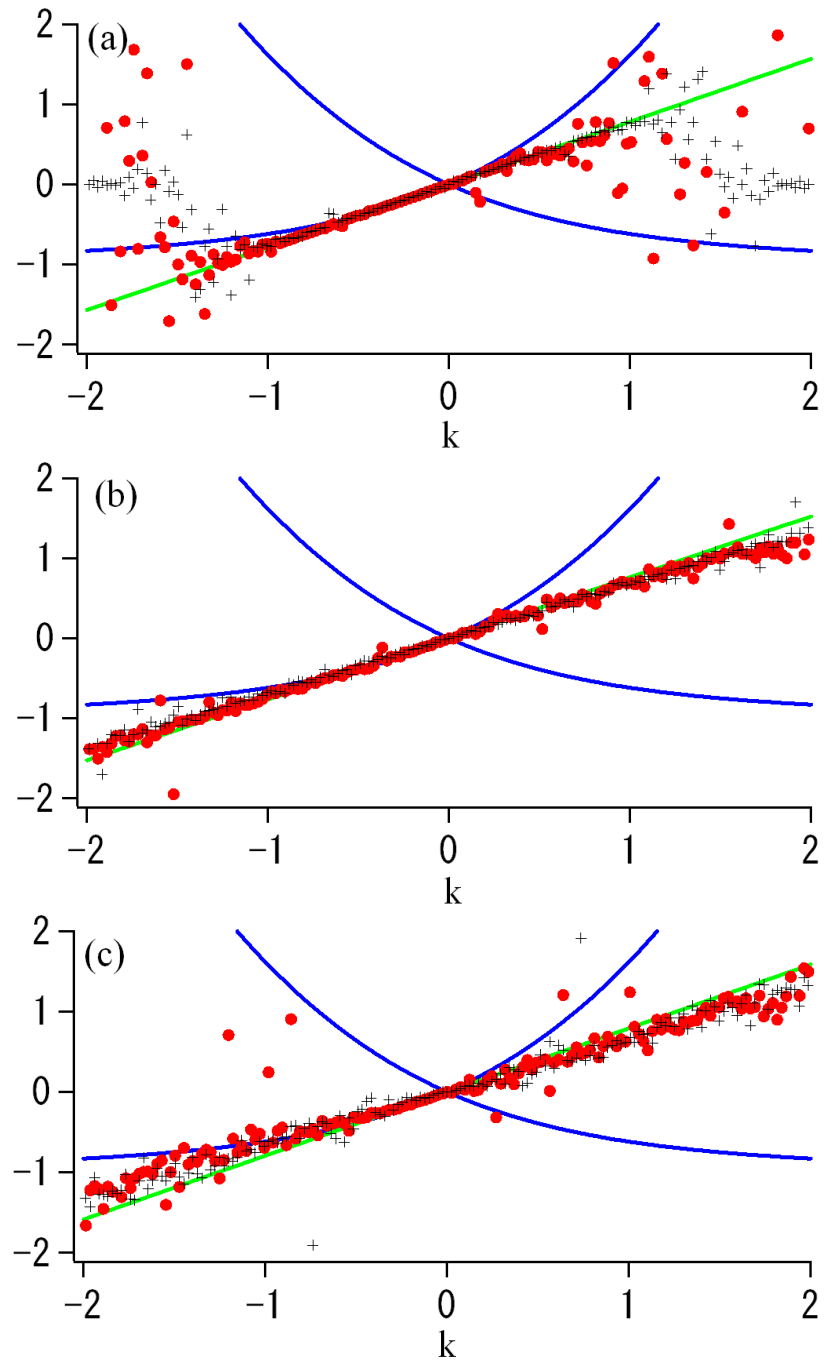

Fig. 13. Numerically obtained dispersion relation at (a) $t=350$, (b) $t=500$, (c) $t=720$. The blue line indicates the linear dispersion relation, and the red circles and black crosses show the frequency evaluated from the temporal variation of the magnetic field mode $\left(\dot{\phi}_{k}^{b}\right)$ and that of the density modes $\left(\dot{\phi}_{k}^{\rho}\right)$, respectively. Superposed (green) line has a slope equal to the phase velocity of the wave mode which has the maximum power. Corresponding to the appearance of solitary waves and localized structures, the wave power tends to be aligned along a straight line.

are artificially removed from the Hall-MHD set of equations. The broken line in Fig. 12a depicts time evolution of the cross correlation function evaluated for the run without the advection terms, using the same simulation parameters as the previous run. The time evolution for both runs are essentially the same, suggesting that the process playing the most dominant role in violating the static approximation is the ponderomotive force, as suggested by Spangler (1987). The self-coupling term cannot be neglected, however, since its magnitude becomes comparable to other terms as the longitudinal wave modes grow.
In a similar way described before to plot the dispersion relation of nonlinear Alfvén waves in the DNLS directly from the simulation run, we have computed the $\dot{\phi}_{k}^{\rho}$ and $\dot{\phi}_{k}^{b}$, using Eqs. (5) and (8), respectively. Corresponding to appearance of solitary waves and localized structures, the frequency distribution $\left(\dot{\phi}_{k}^{\rho}\right.$ and $\left.\dot{\phi}_{k}^{b}\right)$, i.e., the dispersion relation, appears along a straight line, as shown in numerically evaluated dispersion relation, Fig. 13. Since these waves propagate in the same direction as the parent wave, the frequency distribution along a straight line is usually satisfied at the origin. Furthermore, Fig. 13 shows that the longitudinal and transverse wave modes have a similar slope, which is the velocity of the structures composed of these waves. Thus, the three wave resonance condition between the transverse wave modes and the longitudinal wave modes is approximately satisified.

Figure 14 displays time evolution of the wave frequency $\left(\dot{\phi}_{k}^{b}\right)$ for the parent wave $(m=-20$, solid line), and some of the daughter waves ( $m=-8$, dotted line) ( $m=-3$, broken line). When $0<t<\sim 500$, the parent wave mode is dominant, and its frequency stays almost constant. Around $t \sim 500$, the inverse cascade begins to take place, and the parent wave frequency starts to be modified. On the other hand, the frequencies of the lower sideband wave modes remain almost constant (broken line). Thus, the phase velocity of the modes with the maximum power is close to the velocity of the structures, and it corresponds to the slope of the dispersion relation in Fig. 13.

Now we examine the approximation (c) we listed before in the DNLS, i.e., the assumption of constant density in the Hall term. In order to do so, let us explicitly write down the phase relation of Eq. (8),

$\frac{d \phi_{k}^{b}}{d t}=\omega_{n l}+\omega_{\mathrm{disp}}+\omega_{l i n}$,

$\omega_{n l}=k \sum_{k=k 1+k 2} \frac{\left|u_{k 1}\right|\left|b_{k 2}\right|}{\left|b_{k}\right|} \cos \left(\theta_{k=k 1+k 2}^{\mathrm{bub}}\right)$,

$\omega_{\text {disp }}=k_{3} k \sum_{k=k 3+k 4} \frac{\left|b_{k 3}\right|\left|V_{k 4}\right|}{\left|b_{k}\right|} \cos \left(\theta_{k=k 3+k 4}^{\mathrm{bbV}}\right)$,

$\omega_{\text {lin }}=-k \frac{\left|v_{k}\right|}{\left|b_{k}\right|} \cos \left(\phi_{k}^{b}-\phi_{k}^{v}\right)$,

where $V=\rho^{-1}$ and $\theta_{k=l+m}^{x y z}=\phi_{k}^{x}-\phi_{l}^{y}-\phi_{m}^{z}$. In the linear perturbation analysis in Sect. 2.2 we have called $\omega_{n l}, \omega_{\text {disp }}$, and $\omega_{\text {lin }}$ as contribution to the wave frequency via "nonlinear", "dispersion", and "linear" effects, respectively. The dispersion effect is not simply $k^{2}$, but is $k^{2}-b_{0} k_{0} K \rho / 2 / b_{ \pm}^{(*)}$, where the latter term arises due to longitudinal perturbation. Just like in the DNLS model, the balance between the "nonlinear" and the "dispersion" terms also exist in the Hall-MHD equations.

Figure 15 shows $\omega_{n l}, \omega_{\text {disp }}$, and $\omega_{\text {lin }}$ evaluated at $t=350$, 500 , and 720 . We see that $\omega_{\text {lin }}$ is linear in $k$ : this may be 


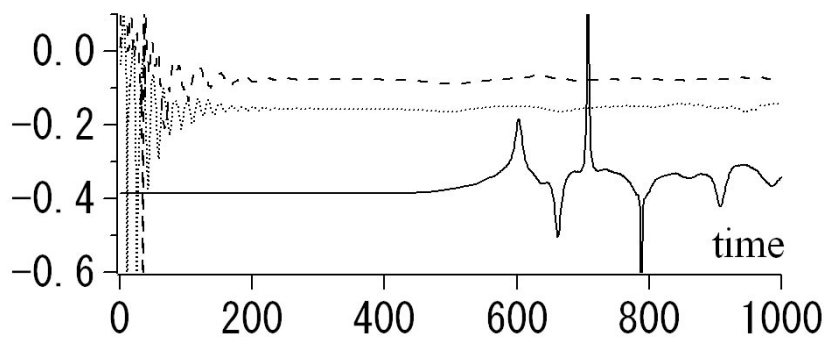

Fig. 14. Time evolution of the wave frequency $\left(\dot{\phi}_{k}^{b}\right)$ for the waves with $m=-20$ (solid line), $m=-8$ (dotted line), and $m=-3$ (broken line). The wave number of these modes are $k=-0.49,-0.19$, and -0.07 , respectively. When $0<t \lesssim 500$, the parent wave mode is dominant, and its frequency is almost constant. Around $t \sim 500$, the inverse cascade begins to take place, and the parent wave frequency starts to vary. On the other hand, the frequencies of the lower sideband wave modes remain almost constant after $t>500$.

regarded as self-generation of the (dispersive) Walen's relation, which is the relation between the transverse magnetic and the velocity field satisfied for Alfvénic fluctuations and rotational discontinuities (e.g., Landau and Lifshitz, 1962). For example, the normalized magnetic and the velocity field, $b_{0}$ and $v_{0}$ in Eqs. (14-15), satisfy $v_{0}=\mp b_{0} / v_{\phi 0}$, where the signs represent parallel/anti-parallel propagation with respect to the background magnetic field, and the phase velocity $v_{\phi 0}$ is determined from the dispersion relation (16). The term $\cos \left(\phi_{k}^{b}-\phi_{k}^{v}\right)$ in the r.h.s. of Eq. (44) takes the value of either 1 or -1 (so that $\omega_{\text {lin }} / k= \pm\left|v_{k}\right| /\left|b_{k}\right|$ ) in association with broadening of the power spectrum. Also, from Figs. 13 and 15 , we find numerically that $\omega_{\operatorname{lin}} / k \sim k / \omega$ (corresponding to the appearance of near straight lines in these plots). Therefore we find that the system approaches automatically to a state where $k / \omega= \pm\left|v_{k}\right| /\left|b_{k}\right|$ (Walen's relation) is automatically fulfilled. Furthermore, since $\omega_{\text {lin }}$ is almost linear in $k$ in Fig. 13, so is the sum of $\omega_{\text {disp }}$ and $\omega_{n l}$ as Eq. (41) suggests, i.e., the contribution to the wave frequency nonlinear in $k$ is cancelled due to balance between the dispersion and the nonlinear effects.

Figure 16 shows distribution of $\dot{\phi}_{k}^{u}$ (similar to $\dot{\phi}_{k}^{\rho}$ ) versus $k$. When $0<t \lesssim 500$, these longitudinal fluctuations are found to be distributed along the same straight line in the dispersion relation, and should be recognized as the ponderomotive density fluctuations, produced via interaction of Alfvén waves. In the present case, the fluctuations are produced in the course of the modulational instability, and the two interacting Alfvén waves are on the same branch with nearly equal wave numbers and the wave frequencies, so that the resultant ponderomotive fluctuations have similar phase velocity as the interacting Alfvén waves. The phase velocities of the longitudinal fluctuations ( $\rho$ and $u$ in Fig. 16), transverse fluctuations ( $b$ and $v$ in Fig. 13), and the acoustic wave (solid line in Fig. 13), are measured as $\sim 0.78, \sim 0.78$, and
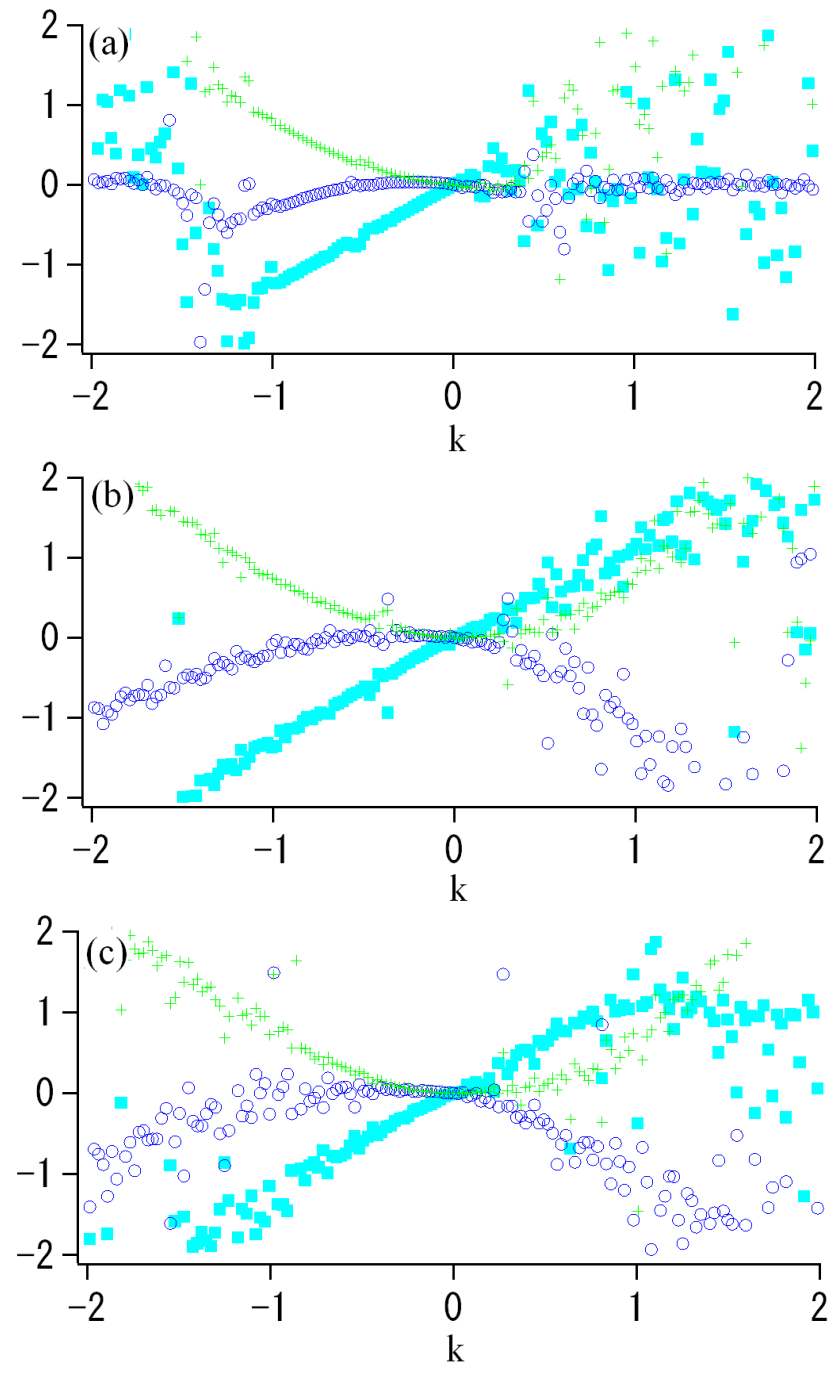

Fig. 15. The three basic elements of the wave frequency, $\omega_{\text {lin }}$ (light blue squares), $\omega_{n l}$ (blue circles), and $\omega_{\text {disp (green crosses), defined }}$ in Eqs. (42-44), plotted versus $k$, evaluated at (a) $t=350$, (b) $t=500$, and (c) $t=720$. As discussed in Sect. 2.2, $\omega_{\text {lin }}$ is almost linear in $k$, suggesting that the dispersive Walen's relation is held. Since the dispersion relation (Fig. 13) suggests that the wave frequency, which is a sum of the three terms above, is linear in $k$ also, the linearity in $k$ of the sum of $\omega_{\text {disp }}$ and $\omega_{n l}$ should be held as well, indicating that the dispersion and the nonlinear effects cancel each other, and the system is brought to a state where the wave resonance condition is easily satisfied.

$\sim 0.70$, respectively. The former two are (almost) equal, and are distinct from the last one. Thus we conclude the longitudinal fluctuations observed at $0<t \lesssim 500$ are the ponderomotive density fluctuations, rather than the sound wave. Around $t \sim 500$, however, the dispersion relations of the longitudinal fluctuaions start to approach the sound wave branch. This is a consequence of the nonlinear development of the modulational instability, in which the ponderomotive density 

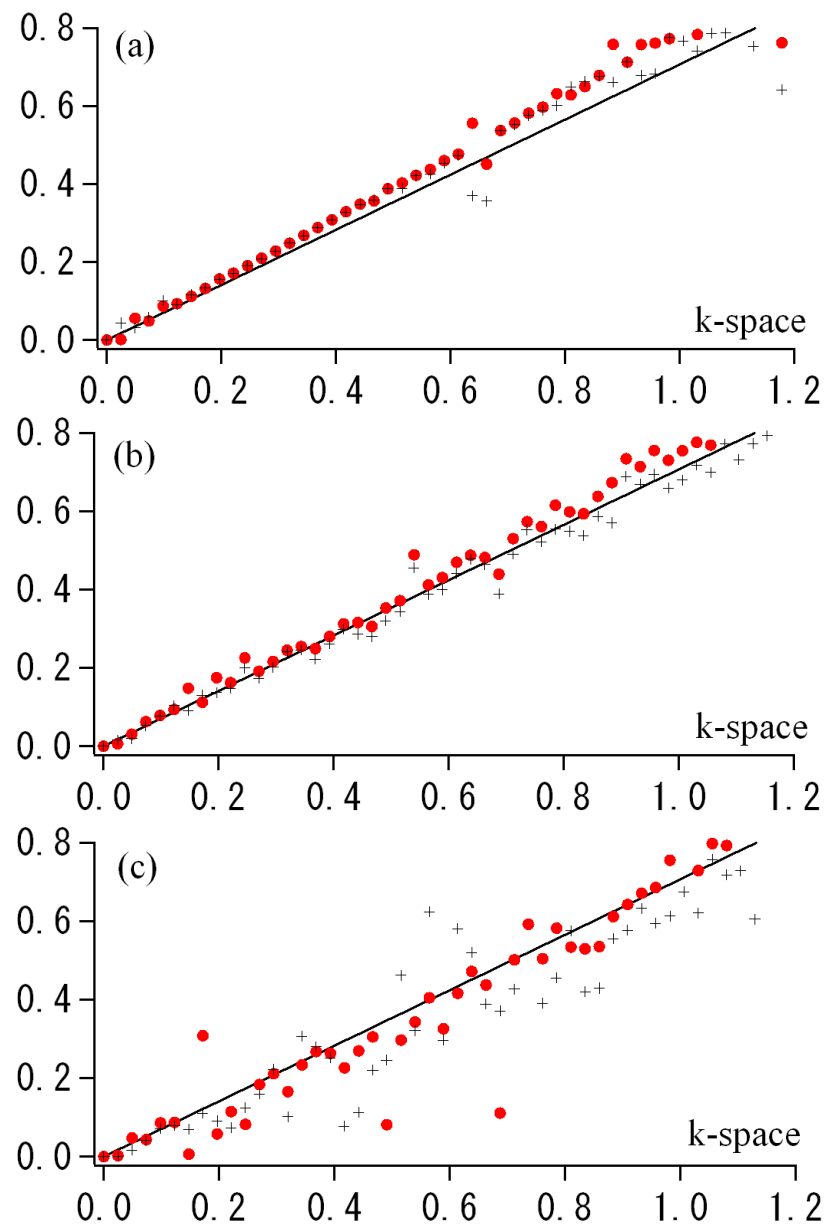

Fig. 16. Numerically computed frequency $\dot{\phi}_{k}^{u}$ (red circles) and $\dot{\phi}_{k}^{\rho}$ (black crosses) versus the wave number $k$ at (a) $\mathrm{t}=350$, (b) $\mathrm{t}=500$, (c) $\mathrm{t}=720$. When $0<t \lesssim 500$, the longitudinal modes $\dot{\phi}_{k}^{u}, \dot{\phi}_{k}^{\rho}$ are distributed around a line slightly (but distinctly) above the sound wave branch (solid black line). The former may be called the "ponderomotive density fluctuation" branch. As later times, however, we find that the longitudinal wave frequencies are re-distributed around the sound branch.

fluctuation acts as a driver to excite the sound waves, as represented in Eq. (40).

This excitation of the sound wave is also related to particle heating due to the modulational instability of Alfvén waves. Many authors discussed Landau damping associated with envelope modulation of Alfvén waves (Mjølhus and Wyller, 1986, 1988; Spangler; 1989, 1990; Medvedev and Diamond, 1996; Passot and Sulem; 2003). However, the ion acoustic wave mode is exclueded, as long as the "ponderomotive" expansion is used in scaling of the system. In the case of the weak nonlinearity, the "triple-degenerate" expansion makes a similar description at $\beta \sim 1$ (Hada, 1993). The kinetic "tripledegenerate" DNLS equation will be useful for elucidating the physics of the heating process. The use of numerical simula- tion models (Vasquez, 1995) and/or theoretical models (Snyder, 1997; Passot and Sulem, 2004) that include the strong nonlinearity and kinetic effects are desired.

\section{Conclusions and discussions}

In this paper, we discussed the nonlinear relation among phases and frequencies in the modulational instability of parallel propagating Alfvén waves in the context of onedimensional two fluid models, using linear perturbation analysis and numerical experiments.

The main results of the present paper may be summarized as follows:

(1) Corresponding to generation of solitary waves or coherent structures by the wave modulation, dispersion relation of the waves tend to be aligned along straight segments of lines (Figs. 7 and 13). The slopes of these straight lines correspond to velocities of the solitary waves and the structures, and are well estimated as the phase ( $\sim$ the group) velocity of the wave with the largest wave power.

(2) The exchange of wave energy among the resonant wave modes is enhanced (reduced) when the relative phase is close to constant (varies rapidly) in time (Fig. 6). This is a universal feature in a wide variety of nonlinear systems: e.g., it can also be seen in a single triplet model (Appendix A).

(3) The fact that the dispersion relation is essentially given as segments of lines in the dispersion relation suggests that the mismatch in the resonance conditions among the coupled wave modes is reduced automatically. The waves can efficiently exchange wave energy and generate the phase coherence.

In the DNLS equation (Sect. 3), increase and decrease of the phase coherence index well corresponds to the appearance and disappearance of solitary wave trains. However, in the Hall-MHD equations (Sect. 4), evolution of the phase coherence index turns out to be more complex. There are two issues to be discussed: the physical processes leading to the variation of the phase coherence index, and the validity of the index.

In the DNLS, the energy flow among the wave modes generates order not only among the wave frequencies but also among wave phases (see Appendix A, and also Fig. 9), and the associated broadening of the power spectrum always corresponds to the global energy flow because of the balance between the dispersion effect and the nonlinear effect. However, in the Hall-MHD, the presence of the localized structures does not always correspond to the broadening of the power spectrum. In Fig. 17 we plot time evolution of $C_{\phi}$ and $b_{\phi}$ based on the Hall-MHD simulation run discussed before. The broadening of the power spectrum correlates well with $b_{\phi}$, but not quite well with $C_{\phi}$. This probably is due to the fact that the structures observed in the simulation are not always generated by the local energy flow. 


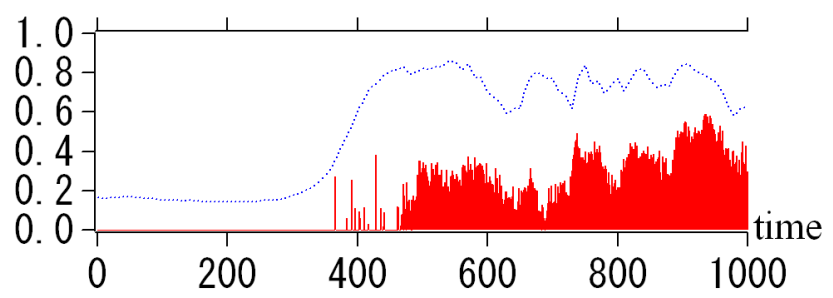

Fig. 17. Time evolution of $C_{\phi}$ (red area) and $b_{\phi}$ (broken line). In association with the broadening of the power spectrum, $b_{\phi}$ varies accordingly, but $C_{\phi}$ does not.

In case of the modulational instability starting from a single parent wave plus small amplitude background noise, one can evaluate the relative phase among the resonant wave modes and also the energy flow among them, and identify clearly time intervals the phase coherence is generated (see Fig. 9, and also early part of Fig. 18). On the other hand, after the initial stage of the modulational instability, wave modes at low wave numbers produced via the inverse cascade become dominant, it is no longer possible to identify the time intervals in which the phase coherence is produced.

Regarding the phase coherence index, we have to say that its physical meaning is still not completely clear, although it was demonstrated that the index is a useful measure to identify the presence of wave-wave interaction (Koga and Hada, 2003). Since the concept of the phase synchronization is universal (e.g., He and Chian, 2005), it is important to develop a method that can characterize the underlying nonlinearity of the system. This discussion is translated into the explanation or improvement of the phase coherence index.

The relation between the longitudinal and transverse modes has important information on the nature of the parametric instability. In the dispersion relation, longitudinal and transverse modes were found to be both linear, with almost the same slope, corresponding to the structures generated by modulational instability. On the other hand, the longitudinal fluctuations generated by the decay instability distribute on the sound wave line with some band width (Terasawa et al., 1986). Such a cross relation among different variables in MHD turbulence is very important to understand the turbulent dynamics. Henceforth, we have to analyze in detail the general rules of the relation among these variables in parallel Alfvén turbulence generated by parametric instability. Also, it is essential to include the kinetic effects when $\beta$ is moderately large.

It is important to identify the phase coherence brought by nonlinear wave-wave interaction, in order to discuss underlying physical processes leading to the generation of the coherent structures. This is particularly important for analysis of spacecraft data, since there are many localized structures in space plasmas which are produced, presumably, by processes other than nonlinear wave-wave interactions. Dudok

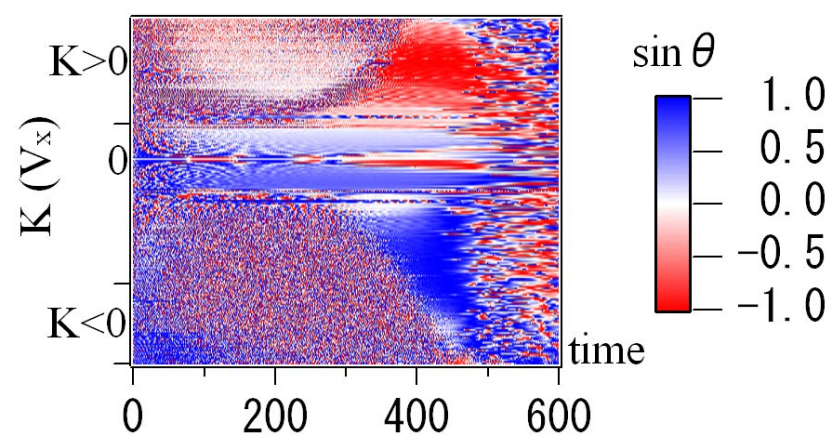

Fig. 18. Time evolution of the relative phase between the parent and the other wave modes, $\sin \left(\phi_{k 0}^{b}-\phi_{k 1}^{b}-\phi_{K}^{u}\right)$.

de Wit et al. (1999) and Soucek et al. (2003) discussed the so-called Volterra model, a way to identify nonlinear wavewave interactions using the satellite and the simulation data, by utilizing the high order spectrum analyses and weak turbulence theory. This method may shed light on understanding the nature of wave-wave interactions when the weak and strong turbulence processes co-exist. In the future, we hope to clarify the process of the phase coherence generation via the wave-wave interaction in more detail, and to extend the Volterra-type models in such a way that the weak turbulence approximation is not necessary.

Finally, we make a remark on energetic particle transport in the turbulence including the localized structures. Since these structures have broad power spectrum in the $k$ space, particles with wide range of energy can resonate with them. However, this is not the more important side of the waveparticle interaction, from the viewpoint of the phase coherence - rather, it is in the fact that large amount of electromagnetic field and plasma energy is concentrated within the solitary wave (phase correlated wave/structure), so that it can influence particle motion via strong and correlated impulse force rather than via sequence of random forces which last for a long time.

For example, let us consider pitch angle diffusion due to the turbulence consisting of superposition of parallel, circularly polarized Alfvén waves (slab model). Within this model, it is well known that the particles cannot diffuse across 90 degrees pitch angle, within the framework of the quasi-linear diffusion, simply because there are no waves which can resonate with particles without parallel velocities. However, the mirror force can reflect the particles quite easily, if there exist solitary waves with a finite amplitude variation of the total magnetic field. Furthermore, in a presence of many of these solitary waves, individual particle trajectory may be given as combination of quasi-ballistic motion between the solitary waves and trapping by one of the solitary waves. Fermi type acceleration is possible (Kuramitsu and Hada, 2000), and furthermore, depending on relative 


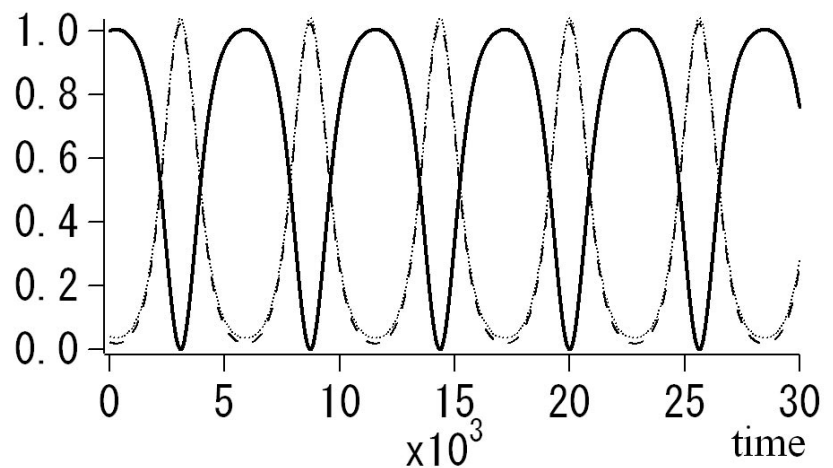

Fig. A1. Time evolution of $N_{j}$ with the initial condition, $N_{3} \gg N_{1}, N_{2}$. The broken, dotted, and solid lines indicate $N_{1}, N_{2}$, and $N_{3}$, respectively. The system is "unstable" in that the "quanta" originally stored in mode 3 are re-distributed to modes 1 and 2 within an instability time scale $(\sim 3000)$.

dominance between the ballistic and trapped trajectories, ensemble of these particles may appear as either super- or subdiffusive (Zimbardo et al., 2000; Carreras et al., 2001). In order to properly describe time evolution of the ensemble, one has to invoke the fractal diffusion formalism (Metzler and Nonnenmacher, 1998; del-Castillo-Negrete et al., 2004).

\section{Appendix A}

\section{Three wave resonance: a single triplet}

A triplet is known as the most basic element of nonlinear interaction that can be extracted from various phenomena. Decay instability of Alfvén waves in space plasma is an example. A finite amplitude Alfvén wave propagating parallel to the ambient magnetic field decays into a backward propagating Alfvén wave and a forward propagating ion acoustic wave. We consider a set of three wave equations (3W) discussed by Sagdeev and Galeev (1969),

$\dot{C}_{1}=-i C_{2}^{*} C_{3}$,

$\dot{C}_{2}=-i C_{1}^{*} C_{3}$,

$\dot{C}_{3}=-i C_{1} C_{2}$,

where $C_{j}$ is the normalized complex amplitude of the $j$-th mode. The above set of equations is derived via resonance condition among frequencies of each mode,

$\omega_{3}=\omega_{1}+\omega_{2}$.

By introducing the wave quanta (wave action), $N_{j}=\left|C_{j}\right|^{2}=\epsilon_{j} / \omega_{j}$, where $\epsilon_{j}$ is the wave energy of mode $j$, we have the Manley-Rowe relations,

$N_{1}+N_{3}=$ const,

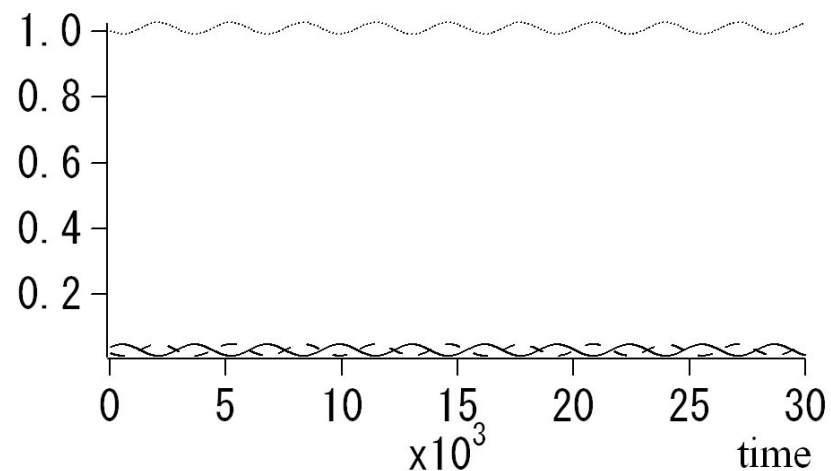

Fig. A2. Same as Fig. A1 except that the initial condition here is $N_{2} \gg N_{1}, N_{3}$. The broken, dotted, and solid lines indicate $N_{1}$, $N_{2}$, and $N_{3}$, respectively. The system is "stable" in the sense that variations of $N_{1}, N_{2}$, and $N_{3}$ are small.

$N_{1}-N_{2}=$ const.

Now let us write $C_{j}=A_{j} \exp \left(i \phi_{j}\right)$, with $A_{j}\left(=\sqrt{N_{j}}\right)$ and $\phi_{j}$ real, then we obtain a relation about the phase difference among the triplets $\left(\theta=\phi_{3}-\phi_{2}-\phi_{1}\right)$,

$$
\begin{aligned}
\dot{\theta} & =\dot{\phi}_{3}-\dot{\phi}_{2}-\dot{\phi}_{1}, \\
& =-\left(\frac{A_{1} A_{2}}{A_{3}}-\frac{A_{2} A_{3}}{A_{1}}-\frac{A_{3} A_{1}}{A_{2}}\right) \cos \theta, \\
& =\cot \theta \frac{d}{d t} \log \left(A_{1} A_{2} A_{3}\right),
\end{aligned}
$$

where $\dot{\phi}_{j}$ is the (nonlinear) frequency and $\dot{\theta}$ represents the frequency mismatch in the resonance condition. From the above, the following conservation law is derived

$A_{1} A_{2} A_{3} \cos \theta=D_{0}=$ const.

The $3 \mathrm{~W}$ equations at a glance have six degrees of freedom, but if we write them into the real amplitude and phase, we find that the equations actually have only four degrees of freedom: the real amplitudes $A_{1}, A_{2}, A_{3}$, and the phase difference $\theta$. Since there are three invariants (A5, A6, A10), the $3 \mathrm{~W}$ equation set is integrable. All initial problem of $3 \mathrm{~W}$ can be solved, and the solution can be written using elliptic functions. Behavior of the system may be physically interpreted as either stable or unstable depending on initial distribution of wave quanta to the three eigenmodes (Figs. A1 and A2).

Let us define the flow of quanta $F$, which represents the interaction between the eigenmodes,

$F=\dot{N}_{1}=\dot{N}_{2}=-\dot{N}_{3}=2 A_{3} A_{2} A_{1} \sin \theta$,

and consider its relationship to temporal change of the phase difference $\dot{\theta}$. The sign of $\sin \theta$ determines the direction of quanta flow.

As for the initial conditions there are two distinct possibilities. The first is that there are some modes without any 


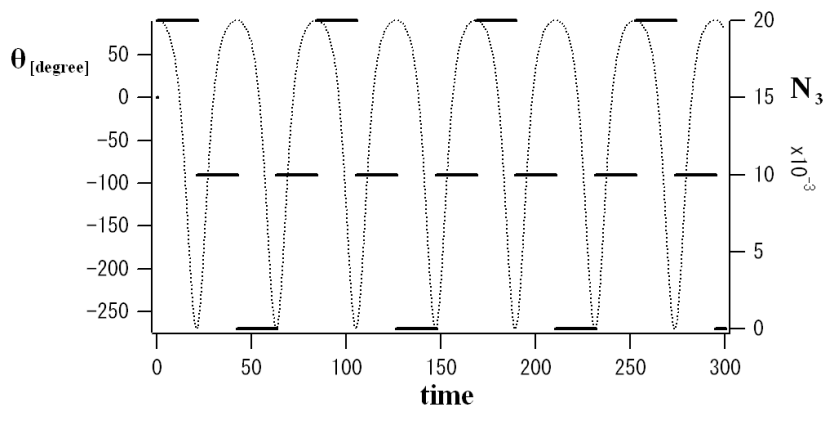

Fig. A3. Time evolution of $N_{1}, N_{2}, N_{3}$, and $\theta$ for the case that $A_{1} A_{2} A_{3} \cos \theta=0$.

quanta, and the second is that all the modes have some finite number of quanta. In the first case, $\cos \theta=0$ always since $D_{0}=0$. In Fig. A3, we see that $\theta$ jumps 180 degrees periodically because the direction of the quanta flow remain unchanged per half the oscillation period.

From now on, we only discuss the case where all the modes have some finite number of quanta. Since $D_{0}=$ cons $\neq 0$, we can rewrite $F$ as

$F=2 D_{0} \tan \theta$

and therefore, the condition $-\pi / 2<\theta<\pi / 2$ is required in order that $F$ be finite. This condition is evident from Eq. (A10). Equation (A12) suggests that $|F|$ is large when $\theta$ is close to either $-\pi / 2$ or $\pi / 2$, while $F=0$ when $\theta=0$ (Fig. A4a).

Figure $\mathrm{A} 4 \mathrm{~b}$ shows time evolution of $\dot{\phi}_{j}$. Both fast and slow temporal changes of $\theta$ can be seen. Around the point where the number quanta is close to take extremal values, the temporal change of $\theta$ becomes rapid. If the system is unstable, temporal change of the phase difference is much faster when the quanta is at local minimum, than when it is at local maximum. Figure A4c shows this relationship more clearly. Using Eqs. (A10) and (A12), and $\dot{\phi_{j}}=-D_{0} / N_{j}$, we have

$\dot{\theta}=-D_{0}\left(\frac{1}{N_{3}}-\frac{1}{N_{1}}-\frac{1}{N_{2}}\right)=D_{0} \frac{\dot{F}}{2 G^{2}}$,

where $G=A_{1} A_{2} A_{3}$. This equation exactly shows the relationship discussed above.

In summary, if the temporal change of the phase difference is small (large), quanta flow between the modes is large (small). In other words, the stronger (weaker) the modes are correlated, are the interaction between the modes strong (weak). This relationship between the phase coherence and the interaction among eigenmodes is universal. For example, similar relationship is held in a system where many triplets are connected, in the DNLS, and in the Hall-MHD models. Also, when $\cos \theta=0$ initially, we have a situation similar to the case that $N_{j}=0$ for one (or more) of the modes, and thus $\cos \theta=0$ always.
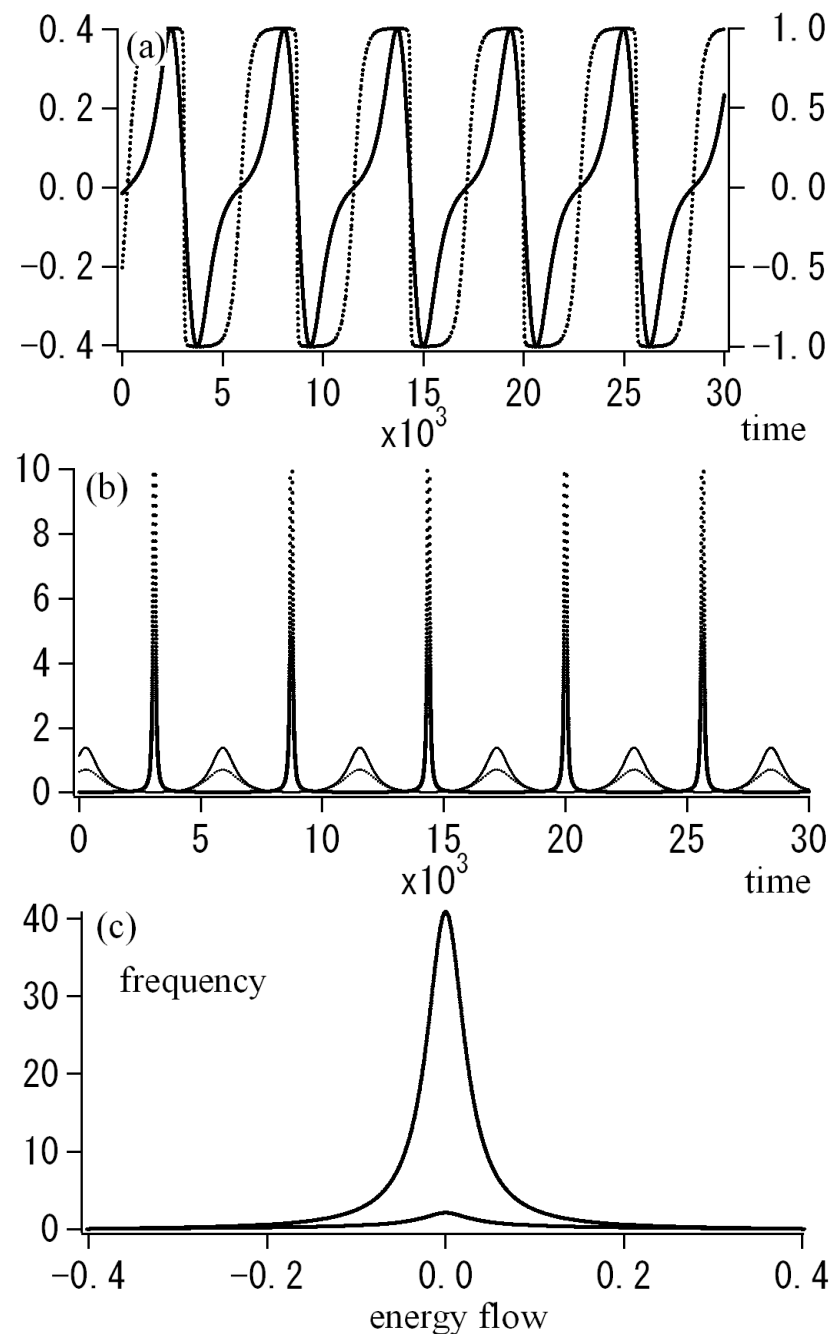

Fig. A4. (a) The energy flow $F$ (solid line, left axis) and $\sin \theta$ (dotted line, right axis) are plotted versus time. When the energy is exchanged between the modes, the relative phase stays at almost at a constant level. The same tendency is observed in systems with multiple degrees of freedom (cf., Figs. 9 and 18). (b) Time evolution of $\dot{\phi}_{j}$. The thin, solid, and dotted lines indicate $\dot{\phi}_{1}, \dot{\phi}_{2}$, and $\dot{\phi_{3}}$, respectively. (c) Relation between $\dot{\theta}$ and $F$ for a (single) triplet. The exchange of quanta between the sites is enhanced when the relative phase is approximately constant in time, while the exchange of quanta is reduced when the relative phase varies rapidly.

Acknowledgements. We thank S. Matsukiyo and D. Koga for fruitful discussions and valuable comments. This paper has been supported by JSPS Research Felowships for Young Scientists in Japan.

Edited by: A. C. L. Chian

Reviewed by: two referees 


\section{References}

del-Castillo-Negrete, D., Carreras, B. A., and Lynch, V. E.: Fractional diffusion in plasma turbulence, Phys. Plasmas, 11(8), 3854-3864, 2004.

Carreras, B. A., Lynch, V, E., and Zaslavsky, G. M.: Anomalous diffusion and exit time distribution of particle tracers in plasma turbulence model, Phys. Plasmas, 8(12), 5096-5103, 2001.

Chanpeaux, S., Laveder, D., Passot, T., and Sulem, P. L. : Remarks on the parallel propagation of small-amplitude dispersive Alfvén waves, Nonlin. Processes Geophys., 6, 169-178, 1999, http://www.nonlin-processes-geophys.net/6/169/1999/.

Chen, X. J. and Lam, W. K.: Inverse scattaring transform for the derivative nonlinear Schrödinger equations with nonvanishing boundary conditions, Phys. Rev. E, 69, 066604, doi:10,1103, 2004.

Derby, N. F.: Modulational instability of finite-amplitude, circulary polarized Alfvén waves, Astrophys. J., 224(3), 1013-1016, 1978.

Diamond, P. H., Rosenbluth, M. N., Sanchez, E., Hidalgo, C., Van Milligen, B., Estrada, T., Branas, B., Hirsch, M., Hartfuss, H. J., and Carreras, B. A.: In seach of the elusive zonal flow using cross-bicoherence analysis, Phys. Rev. Lett., 84(21), 4842-4845, 2000.

Dudok de Wit, T., Krasnosel'skikh, V. V., Dunlop, M., and Luhr, H.: Identifying nonlinear wave interactions in plasmas using twopoint measurements: A case study of Short Large Amplitude Magnetic Structures (SLAMS), J. Geophys. Res., 104, 17079 $17090,1999$.

Galeev, A. A., Oraevskii, V. N., and Sagdeev, R. Z.: Universal instability of an inhomogeneous plasma in a magnetic feild, Sov. Phys. JETP, 17, 615-620, 1963.

Ghosh, S. and Papadopoulos, K.: The onset of Alfvénic turbulence, Phys. Fluids, 30(5), 1371-1387, 1987.

Goldstein, M. L.: An instability of finite amplitude circularly polarized Alfvén waves, Astrophys. J., 219(2), 700-704, 1978.

Hada, T.: Evolution of large amplitude Alfvén waves in the solar wind with $\beta \sim 1$, Geophys. Res. Lett., 20(22), 2415-2418, 1993.

Hada, T., Koga, D., and Yamamoto, E.: Phase coherence of MHD waves in the solar wind, Space Sci. Rev., 107(1-2), 463-466, 2003.

Hasegawa, A., Kodama, Y., and Watanabe, K.: Self-organization in Korteweg - de Vries turbulence, Phys. Rev. Lett., 47(21), 1525$1529,1981$.

He, K. and Chian, A. C.-L.: Nonlinear dynamics of turbulent waves in fluids and plasmas, Nonlin. Processes Geophys., 12, 13-24, 2005 , http://www.nonlin-processes-geophys.net/12/13/2005/.

Hollweg, J. V.: Beat, modulational, and decay instabilities of a circularly polarized Alfvén wave, J. Geophys. Res, 99, 23431 23 447, 1994.

Kaup, D. J. and Newell, A. C.: An exact solution for a derivative nonlinear Schrödinger equation, J. Math. Phys., 19, 798-801, 1978.

Kawata, T. and Inoue, H.: Exact solution of the derivative nonlinear Schrödinger equation under the nonvanishing conditions, J. Phys. Soc. Japan, 44, 1968-1976, 1978.

Kawata, T., Sakai, J.-I., and Kobayashi, N.: Inverse method for the mixed nonlinear Schrödinger equation and soliton solutions, J. Phys. Soc. Japan, 48, 1371-1379, 1980.

Koga, D. and Hada, T.: Phase coherence of foreshock MHD waves: wavelet analysis, Space. Sci. Rev., 107, 495-498, 2003.

Krishan, V. and Nocera, L.: Relaxed states of Alfvénic turbulence, Phys. Lett. A, 315, 389-394, 2003.

Kuramitsu, Y. and Hada, T.: Acceleration of charged particles by large amplitude MHD waves: effect of wave spatial correlation, Geophys. Res. Lett., 27(5), 629-632, 2000.

Landau, L. D. and Lifshitz, E. M.: Electrodynamics of continuous media, Pergamon, Oxford, New York, 1984.

Longtin, M. and Sonnerup, B.: Modulational instability of circulary polarized Alfvén waves, J. Geophys. Res., 91, 798-801, 1986.

Lucek, E. A., Horbury, T. S., Balogh, A., Dandouras, I., and Reme, H.: Cluster obsevations of structures at quasi-parallel bow shocks, Ann. Geophys., 22, 2309-2314, 2004, http://www.ann-geophys.net/22/2309/2004/.

Machida, S., Spangler, S. R., and Goertz, C. K.: Simulation of amplitude-modulated circularly polarized Alfvén waves for beta less than one, J. Geophys. Res., 92(A7), 7413-7422, 1987.

Mann, G., Luhr, H., and Baumjohann, W.: Statistical analysis of short large-amplitude magnetic field structures in the vicinity of the quasi-parallel bow shock, J. Geophys. Res., 99(A7), 13315$13323,1994$.

Medvedev, M. D. and Diamond, P. H.: Fluid models for kinetic effects on coherent nonlinear Alfvén waves, Phys. Plasmas, 3(3), 863-873, 1996.

Medvedev, M. D., Diamond, P. H., Shevchenko, V. I., and Galinsky, V. L.: Dissipative dynamics of collisionless nonlinear Alfvén wave trains, Phys. Rev. Lett., 78(26), 4934-4937, 1997.

Metzler, R. and Nonnenmacher, T. F.: Fractional diffusion, waitingtime distributions, and Cattaneo-type equations, Phys. Rev. E, 57(6), 6409-6414, 1998.

Mio, K., Ogino, T., Minami, K., and Takeda, S.: Modified nonlinear Schrödinger equation for Alfvén waves propagating along the magnetic field in cold plasmas, J. Phys. Soc. Japan, 41, 265-271, 1976.

Mjølhus, E.: Application of the reductive perturbation method to long hydromagnetic waves parallel to the magnetic field in a cold plasma, Report no. 48, Department of applied mathematics, Univ. Bergen, Norway, 1974.

Mjølhus, E.: On the modulational instability of hydromagnetic waves parallel to the magnetic field, J. Plasma. Phys., 16, 321334, 1976.

Mjølhus, E. and Wyller, J.: Alfvén solitons, Phys. Scr., 33, 442451, 1986.

Mjølhus, E. and Wyller, J.: Nonlinear Alfvén waves in a finite beta plasma, J. Plasma Phys., 40, 299-318, 1988.

Mjølhus, E. and Hada, T.: Soliton theory of quasi-parallel Alfvén waves, in: Nonlinear waves and chaos in space plasmas, edited by: Hada, T. and Matsumoto, H., Terrapub, Tokyo, 121-169, 1997.

Nariyuki, Y. and Hada, T.: Self-generation of phase coherence in parallel Alfvén turbulence, Earth, Planets, Space, 57, e9-e12, 2005.

Nocera, L. and Buti, B.: Bifurcations of coherent states of the DNLS equation, Phys. Scr., T63, 186-189, 1996.

Passot, T. and Sulem, P. L.: Long-Alfvén wave trains in collisionless plasmas. 1. Kinetic theory, Phys. Plasmas, 10(10), 38873905, 2003.

Passot, T. and Sulem, P. L.: A Landau fluid model for dispersive magnetohydrodynamics, Phys. Plasmas, 11(11), 5173- 
5189,2004.

Rogister, A.: Parallel propagation of nonlinear low-frequency waves in high-beta plasma, Phys. Fluids, 14, 2733-2739, 1971.

Sagdeev, R. Z. C. and Galeev, A. A.: Nonlinear Plasma Theory, edited by: O’Neil, T. M., Book, D. L., and Benjamin, W. A., New York, 1969.

Sakai, J.-I. and Sonnerup, B.: Modulational instability of finite amplitude dispersive Alfvén waves, J. Geophys. Res., 88, 90699078, 1983.

Snyder, P. B., Hammett, G. W., and Dorland, W.: Landau fluid models of collisionless magnetohydrodynamics, Phys. Plasmas, 4(11), 3974-3985, 1997.

Soucek, J., Dudok de Wit, T., Krasnoselskikh, V., and Volokitin, A.: Statical analysis of nonlinear wave interactions in simulated Langmuir turbulence data, Ann. Geophys., 21, 681-692, 2003, http://www.ann-geophys.net/21/681/2003/.

Spangler, S. R. and Sheerin, J. P.: Properties of Alfvén solitons in a finite-beta plasma, J. Plasma Phys., 27, 193-198, 1982.

Spangler, S. R.: The evolution of nonlinear Alfvén waves subject to growth and damping, Phys. Fluids, 29(8), 2535-2547, 1986.

Spangler, S. R.: Density fluctuations induced by nonlinear Alfvén waves, Phys. Fluids, 30(4), 1104-1109, 1987.

Spangler, S. R.: Kinetic effects on Alfvén-wave nonlinearity, 1. Ponderomotive density fluctuations, Phys. Fluids, B 1(8), 17381746, 1989.

Spangler, S. R.: Kinetic effects on Alfvén-wave nonlinearity, 2. The modified nonlinear-wave equation, Phys. Fluids, B 2(2), 407418, 1990.
Terasawa, T., Hoshino, M., Sakai, J.-I., and Hada, T.: Decay instability of finite-amplitude circulary polarized Alfvén waves: A numerical simulation of stimulated brillouin scattering, J. Geophys. Res., 91, 4171-4187, 1986.

Tsurutani, B. T., Lakhina, G. S., Pickett, J. S., Guarnieri, F. L., Lin, N., and Goldstein, B. E.: Nonlinear Alfvén waves, proton perpendicular acceleration, and magnetic holes/decreases in interplanetary space and the magnetosphere: intermediate shocks?, Nonlin. Processes Geophys., 12, 321-336, 2005, http://www.nonlin-processes-geophys.net/12/321/2005/.

Vasquez, B. J.: Simulation study of the role of ion kinetics in low-frequency wave train evolution, J. Geophys. Res., 100(A2), 1779-1792, 1995.

Vinaz, A. F. and Goldstein, M. L.: Parametric instabilities of circularly polarized large-amplitude dispersive Alfvén waves: excitation of parallel-propagating electromagnetic daughter waves, J. Plasma. Phys., 46, 107-127, 1991.

Wong, H. K. and Goldstein, M. L.: Parametric instabilities of circularly polarized Alfvén waves including dispersion, J. Geophys. Res., 91, 5617-5628, 1986.

Zimbardo, G., Greco, A., and Veltri, P.: Superballistic transport in tearing driven magnetic turbulence, Phys. Plasmas, 7(4), 1071$1074,2000$. 